\title{
INTERACTING GALAXIES IN THE A901/902 SUPERCLUSTER WITH STAGES
}

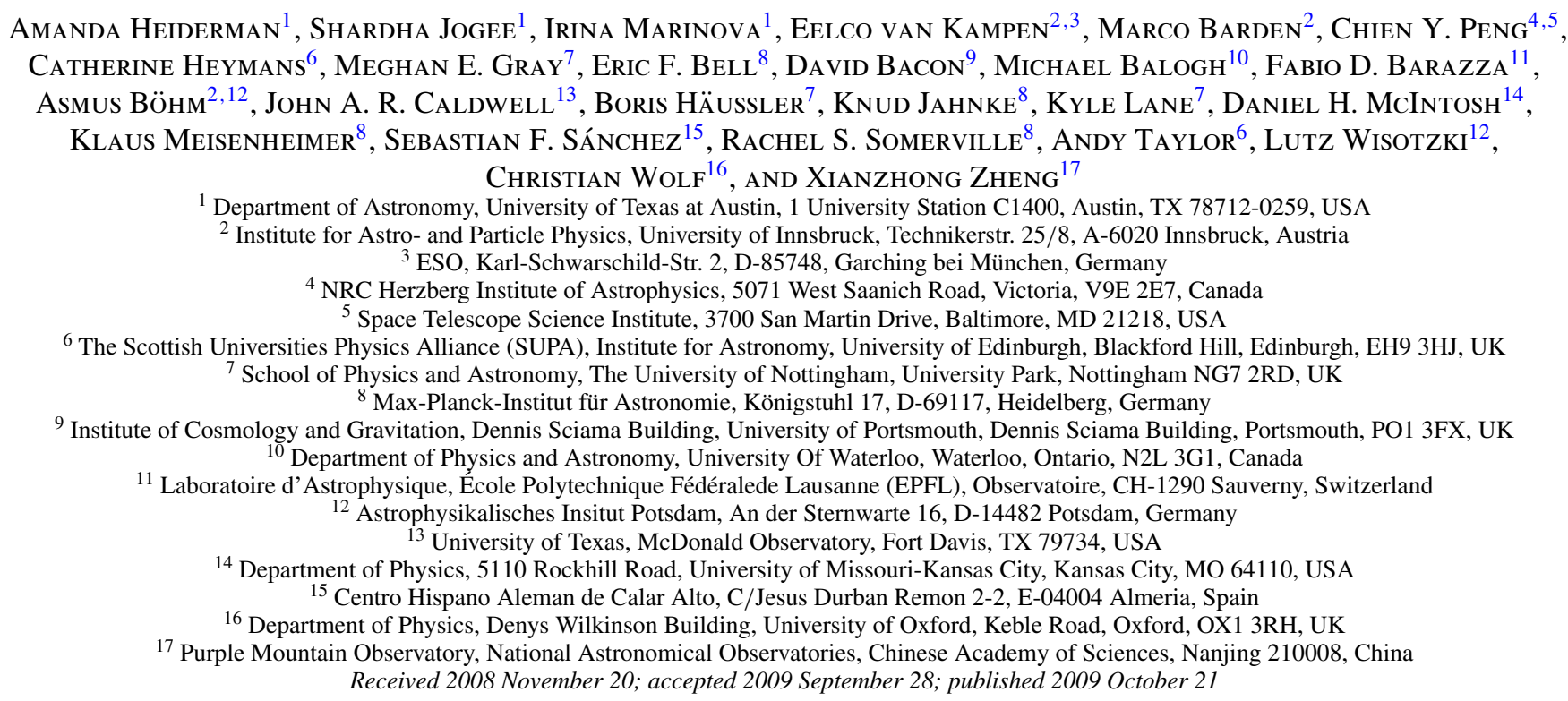

\section{ABSTRACT}

We present a study of galaxy mergers and the influence of environment in the Abell 901/902 supercluster at $z \sim 0.165$, based on 893 bright $\left(R_{\text {Vega }} \leqslant 24\right)$ intermediate-mass $\left(M_{*} \geqslant 10^{9} M_{\odot}\right)$ galaxies. We use $H S T$ ACS F606W data from the Space Telescope A901/902 Galaxy Evolution Survey, COMBO-17, Spitzer $24 \mu \mathrm{m}$, and $X M M$-Newton X-ray data. Our analysis utilizes both a physically driven visual classification system and quantitative CAS parameters to identify systems which show evidence of a recent or ongoing merger of mass ratio $>1 / 10$ (i.e., major and minor mergers). Our results are (1) after visual classification and minimizing the contamination from false projection pairs, we find that the merger fraction $f_{\text {merge }}$ is $0.023 \pm 0.007$. The estimated fractions of likely major mergers, likely minor mergers, and ambiguous cases are $0.01 \pm 0.004,0.006 \pm 0.003$, and $0.007 \pm 0.003$, respectively. (2) All the mergers lie outside the cluster core of radius $R<0.25 \mathrm{Mpc}$ : the lack of mergers in the core is likely due to the large galaxy velocity dispersion in the core. The mergers, instead, populate the region $(0.25 \mathrm{Mpc}<R \leqslant 2 \mathrm{Mpc})$ between the core and the cluster outskirt. In this region, the estimated frequency of mergers is similar to those seen at typical group overdensities in $N$-body simulations of accreting groups in the A901/902 clusters. This suggests the ongoing growth of the clusters via accretion of group and field galaxies. (3) We compare our observed merger fraction with those reported in other clusters and groups out to $z \sim 0.4$. Existing data points on the merger fraction for $L \leqslant L^{*}$ galaxies in clusters allow for a wide spectrum of scenarios, ranging from no evolution to evolution by a factor of $\sim 5$ over $z \sim 0.17-0.4$. (4) In A901/902, the fraction of interacting galaxies, which lie on the blue cloud is $80 \% \pm 18 \%(16 / 20)$ versus $34 \% \pm 7 \%$ or $(294 / 866)$ for non-interacting galaxies, implying that interacting galaxies are preferentially blue. (5) The average star formation rate (SFR), based on UV or a combination of UV+IR data, is enhanced by a factor of $\sim 1.5-2$ in mergers compared to non-interacting galaxies. However, mergers in the A901/902 clusters contribute only a small fraction (between $10 \%$ and 15\%) of the total SFR density, while the rest of the SFR density comes from non-interacting galaxies.

Key words: galaxies: clusters: general - galaxies: clusters: individual (A901, A902) - galaxies: evolution galaxies: formation - galaxies: interactions - galaxies: structure

Online-only material: color figures

\section{INTRODUCTION}

Understanding how galaxies evolve in various environments (field, groups, and clusters), and as a function of redshift is a key step toward developing a coherent picture of galaxy evolution. Present-day cluster and field galaxies differ due to several factors, which are often grouped under the umbrella of "nature" versus "nurture." First, in cold dark matter (CDM) cosmogonies, the first galaxies formed and evolved early in cluster cores, as the higher initial overdensities led to faster gravitational collapse and more rapid mergers of proto-galaxies (e.g., Cole et al. 2000; Steinmetz \& Navarro 2002). Second, in the context of the bottom-up CDM assembly paradigm, the outer parts of clusters and superclusters grow at late times via mergers, smooth accretion, and discrete accretion of groups and field galaxies. This idea is supported by observational studies (e.g., Zabludoff \& Franx 1993; Abraham et al. 1996; Balogh et al. 2000), which suggest that clusters continuously grew by the accretion 
of groups. Third, the dominant physical processes affecting galaxies differ in cluster and field environments due to the different galaxy number density, galaxy velocity dispersion, and intracluster medium (ICM). Among these processes are close galaxy-galaxy interactions, such as strong tidal interactions and mergers (e.g., Barnes 1992; Moore et al. 1998) and galaxy harassment (e.g., Moore et al. 1996), which stems from the cumulative effect of weak interactions. Furthermore, in clusters where the hot ICM makes up as much as $15 \%$ of the total mass, galaxy-ICM interactions, such as ram pressure stripping (Gunn \& Gott 1972; Larson et al. 1980; Quilis et al. 2000; Balogh et al. 2000), can play an important role in removing the diffuse gas from galaxies. The tidal field of the cluster potential may also play a relevant role in the dynamical evolution of cluster galaxies (Gnedin 2003).

Systematic studies of the differences between cluster, group, and field galaxies at different redshifts are needed to shed light on the relative importance of these various processes in their respective environments. Several differences have been observed between galaxies in the field and those in the rich cluster environment, but the physical drivers behind these variations are still under investigation. At $z \sim 0$, the relative percent of massive early-type (E+S0) galaxies to spirals rises from $(10 \%+10 \%: 80 \%)$ in the field to $(40 \%+50 \%: 10 \%)$ in the cores of very rich clusters, leading to the so-called morphology density relation (Dressler 1980; Dressler et al. 1997). However, recent Sloan Digital Sky Survey (SDSS) studies suggest that masses and star formation (SF) histories of galaxies are more closely related to environmental physical processes rather than their structural properties (Blanton et al. 2005). The SF histories of galaxies depend on both luminosity (Cole et al. 2001) and environment (Diaferio et al. 2001; Koopmann \& Kenney 2004). The fraction of blue galaxies in clusters appears to rise with redshift, an effect known as the Butcher Oemler effect (Butcher \& Oemler 1978; Margoniner et al. 2001; de Propis et al. 2003). There is also evidence that SF in bright $\left(M_{\mathrm{v}}<-18\right)$ cluster galaxies is suppressed compared to field galaxies (e.g., Balogh et al. 1998, 1999), for reasons that are not well understood.

Galaxy interactions and mergers have been proposed as a mechanism for the change in galaxy populations in clusters from that of the field (e.g., Toomre \& Toomre 1972; Lavery \& Henry 1988; Lavery et al. 1992). There have been various studies on the properties of galaxies (e.g., Dressler 1980; Postman \& Geller 1984; Giovanelli et al. 1986; Kennicutt 1983; Gavazzi \& Jaffe 1985; Whitmore et al. 1993) and of galaxy interactions and mergers (e.g., Lavery \& Henry 1988; Lavery et al. 1992; Zepf et al. 1993; Dressler et al. 1994; Couch et al. 1998; van Dokkum et al. 1998, 1999; Tran et al. 2005, 2008) in different environments. Some of these studies suggest that galaxy interactions and mergers may play a role in morphological transformations of galaxies in clusters, but there have been few systematic studies of galaxy mergers and interactions in clusters, based on high-resolution Hubble Space Telescope (HST) images as well as Spitzer $24 \mu \mathrm{m}$, and X-ray images.

In this paper, we present a study of the frequency, distribution, color, and SF properties of galaxy mergers in the A901/ 902 supercluster at $z \sim 0.165$. We use HST ACS F606W data taken as part of the Space Telescope A901/902 Galaxy Evolution Survey (STAGES; Gray et al. 2009), along with ground-based COMBO-17 imaging data (Wolf et al. 2004), Spitzer $24 \mu \mathrm{m}$ data (Bell et al. 2005, 2007), XMM-Newton X-ray data (Gilmour et al. 2007), and dark matter (DM) mass mea- surements from weak lensing (Heymans et al. 2008). With a resolution of $0^{\prime \prime} .1$ or $\sim 280 \mathrm{pc}$ at $z=0.165$, the $H S T$ images allow for the identification of merger signatures such as double nuclei, arcs, shells, tails, tidal debris, and accreting satellites. The COMBO-17 survey (Wolf et al. 2004) provides accurate spectrophotometric redshifts down to $R_{\text {Vega }}$ of 24 and stellar masses (Borch et al. 2006). The Spitzer $24 \mu \mathrm{m}$ data (Bell et al. 2005, 2007) probe the obscured SF, while X-ray maps (Gilmour et al. 2007; M. E. Grey et al. 2010, in preparation) provide information of how the ICM density changes throughout the cluster.

We present the data and sample selection in Section 2. In Sections 3.2 and 3.4, we describe the two different methods that we use to identify galaxy mergers: a physically motivated classification system that uses visual morphologies, stellar masses, and spectrophotometric redshifts, and a method based on CAS asymmetry $A$ and clumpiness $S$ parameters (Conselice et al. 2000). In Sections 4.1 and 4.2, we explore the frequency of galaxy mergers in A901/902 based on these two methods and present one of the first systematic comparisons to date between CAS-based and visual classification results in clusters. We set a lower limit on the fraction of major mergers (those with mass ratio $\left.M_{1} / M_{2} \geqslant 1 / 4\right)$. In Section 4.3 , we examine the distribution of mergers in the A901/902 supercluster as a function of clustocentric radius, galaxy number density, local galaxy surface density, relative ICM density, and local DM mass surface density. In Section 4.4, we compare our results on the fraction and distribution of mergers to expectations based on analytical estimates and simulations of mergers in different environments. In Section 4.5, we compare our results on galaxy mergers in the A901/902 supercluster to groups and clusters at different redshifts out to $z \sim 0.8$. We investigate the fraction of mergers and non-interacting galaxies on the blue cloud and red sample as a function of clustocentric radius in Section 4.6. Finally in Section 4.7, we compare the star formation rate (SFR) of mergers and non-interacting galaxies in the A901/902 clusters. The results of this work are summarized in Section 5. In this paper, we assume a flat cosmology with $\Omega_{m}=1-\Omega_{\lambda}=$ 0.3 and $H_{0}=70 \mathrm{~km} \mathrm{~s}^{-1} \mathrm{Mpc}^{-1}$ throughout this paper.

\section{THE A901/902 SUPERCLUSTER: DATA SET AND SAMPLE SELECTION}

The A901/902 supercluster is composed of three clusters: A901a, A901b, and A902, and related groups (Gray et al. 2002; Heymans et al. 2008). This study utilizes data from the STAGES survey (Gray et al. 2009), which provides high-resolution F606W Hubble Space Telescope (HST) Advanced Camera for Surveys (ACS) images over a $0.5 \times 0.5$ field. Additional multiwavelength data include XMM-Newton (Gilmour et al. 2007), Spitzer $24 \mu \mathrm{m}$ data (Bell et al. 2005, 2007), and ground-based COMBO-17 imaging data (Wolf et al. 2004).

Stellar masses are taken from Borch et al. (2006). They were derived by fitting the 17-band COMBO spectral energy distributions (SEDs) with a library of template SEDs, which were constructed using the PEGASE stellar population synthesis model, assuming different SF histories and a Kroupa (Kroupa et al. 1993) initial mass function (IMF) in the mass regime 0.1$120 M_{\odot}$. Such stellar masses are consistent within $10 \%$ with masses estimated using a Kroupa (2001) or Chabrier (2003) IMF. $^{18}$

\footnotetext{
18 We adopt a Chabrier (2003) IMF when exploring the contribution of mergers to the SFR in Section 4.7.
} 


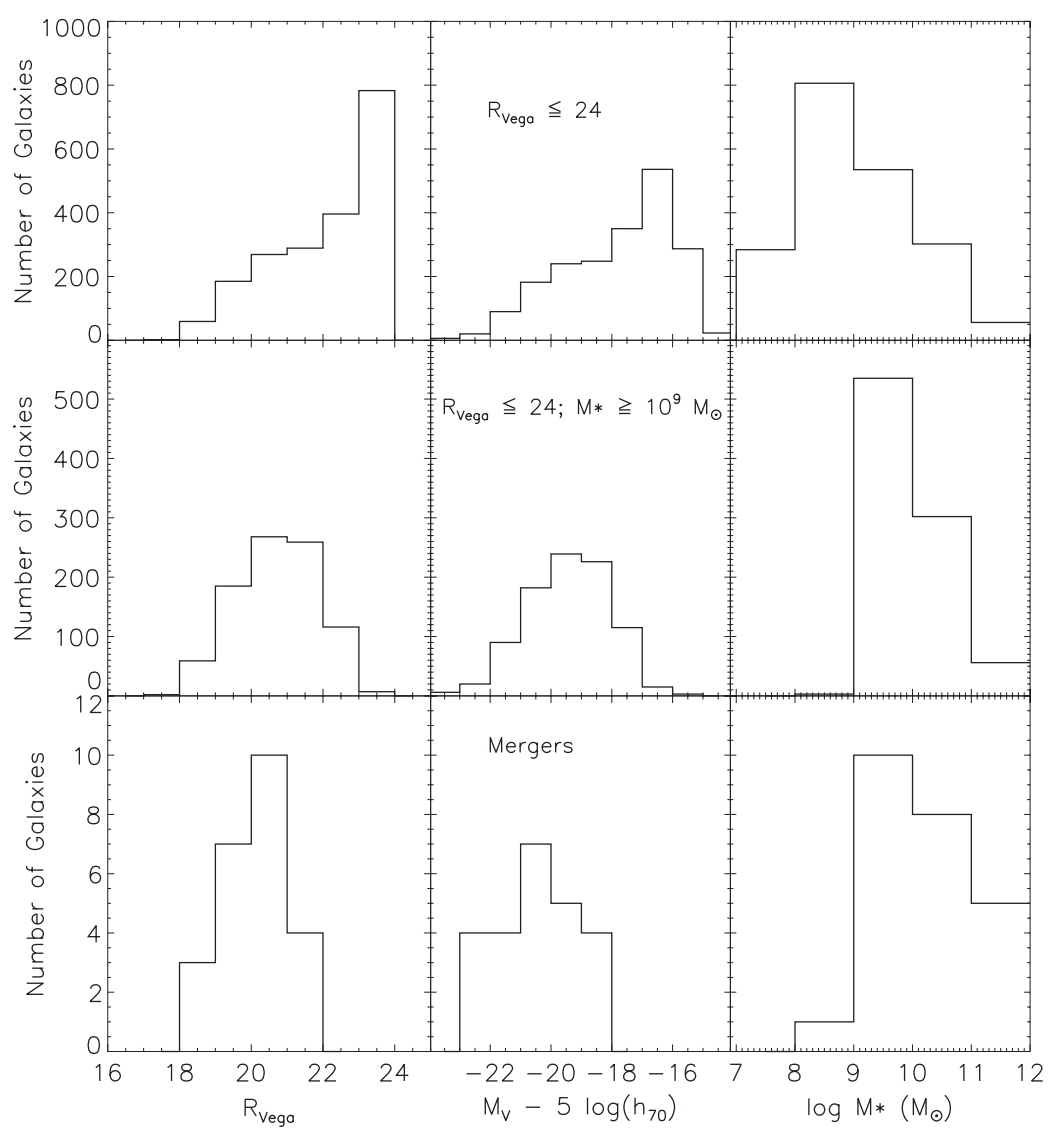

Figure 1. Top panel: apparent $R$-band magnitude $\left(R_{\mathrm{Vega}}\right)$, absolute visual magnitude $\left(M_{\mathrm{V}}\right)$, and stellar mass $\left(M_{*}\right)$ distributions of the full A901/902 supercluster sample $\left(R_{\text {Vega }} \leqslant 24 ; 1983\right.$ galaxies). Middle panel: same as the top panel, but showing the properties of the sample of 893 intermediate-mass galaxies $\left(M_{*} \geqslant 10^{9} M_{\odot}\right)$. Bottom panel: same as the middle panel, but only showing the properties of the 24 galaxies, which are part of the 20 morphologically distorted mergers identified among the sample (see Section 3.2 and Table 2 for details). These 20 mergers are labeled with a starred identification number in Table 2 and are Cases 1-13, 16-18, $20,24,34,35$ in Table 2. For the four mergers of Type $2 \mathrm{~b}$ (which are close pairs resolved by COMBO-17) we plot the properties of the eight individual galaxies making up the pair, thus resulting in a total of 24 galaxies. Each pair has a galaxy of mass $M_{*} \geqslant 10^{9} M_{\odot}$ and a second galaxy that may be of any mass.

Accurate spectrophotometric redshifts down to $R_{\mathrm{Vega}} \sim 24$ and SEDs, based on 5 broad bands (UBVRI) and 12 medium band filters, are available from the COMBO-17 project (Wolf et al. 2004). The $1 \sigma$ redshift error $\left(\sigma_{z(R)}\right)$ modeled from spectroscopic redshifts that are available for a subset of 420 galaxies, scales as

$$
\frac{\sigma_{z(R)}}{(1+z)}=0.005 \times \sqrt{1+10^{0.6(R-20.5)}}
$$

where $R$ is the apparent $R$-band magnitude of the galaxy measured in a $1^{\prime \prime} .5$ aperture.

The reduction of the ACS images and the selection of the A901/902 supercluster sample are described in detail in Gray et al. (2009), and we only provide a summary here. STAGES A901/902 object detection was automated using source extractor (SExtractor; Bertin \& Arnouts 1996) on the ACS F606W images, yielding $\sim 12,500$ galaxies with matching COMBO-17 counterparts down to $R_{\text {Vega }} \sim 24$. A subset of these galaxies was considered to be supercluster members based on their spectrophotometric redshifts. The spectrophotometric redshifts of cluster members were assumed to follow a Gaussian distribution with a half-width $(\Delta z)$ related to $\sigma_{z(R)}$. A galaxy is considered to be a cluster member if its spectrophotometric redshift $z$ lies in the range $[0.17-\Delta z, 0.17+\Delta z]$, where

$$
\Delta z(R)=\sqrt{0.015^{2}+\left(1.65 \sigma_{z(R)}\right)^{2}} .
$$

The resulting dependence of the half-width $\Delta z$ on $R$ ensures a completeness level of $>90 \%$ at all magnitudes for $R_{\text {Vega }} \leqslant$ 24. The field contamination (i.e., the fraction of galaxies that are field members) for this sample is estimated by assuming that the average number count of field galaxies as a function of magnitude and redshift is consistent with the trends determined from regions lying outside of the cluster. The amount of field contamination rises strongly at fainter magnitude due to the increase of $\sigma_{z(R)}$ at fainter $R$, and typical values are $20 \%$ at $R_{\text {Vega }}=21.65$ and $\sim 70 \%$ at $R_{\text {Vega }}=24$ (see Figures 13 and 14 of Gray et al. 2009).

The top panel of Figure 1 shows the distribution of apparent $R$-band magnitude, absolute magnitude $M_{\mathrm{V}}$, and stellar mass for the sample of 1990 galaxies with $R_{\mathrm{Vega}} \leqslant 24$. We obtain our working sample of 893 galaxies by applying a further cut of $M_{*} \geqslant 10^{9} M_{\odot}$ for the following reasons. First, for $M_{*} \geqslant 10^{9} M_{\odot}$, the sample is complete in stellar mass on the red cloud and blue sequence (Borch et al. 2006), while at lower masses incompleteness sets in. Second, as shown in the middle panel of Figure 1, most galaxies with $R_{\text {Vega }} \leqslant 24$ and $M_{*} \geqslant 10^{9} M_{\odot}$ actually have apparent magnitudes of $R_{\text {Vega }} \leqslant$ 21.7 , where the field contamination is $\leqslant 20 \%$ (see Figures 13 and 14 of Gray et al. 2009). Third, most galaxies with $M_{*} \geqslant 10^{9} M_{\odot}$ have a luminosity $M_{\mathrm{V}} \leqslant-18$ (Figure 1), where the cluster luminosity function (Binggeli et al. 1988) is typically dominated by E to Sd galaxies, rather than small dwarf galaxies. The former galaxies are large and many of their morphological features 
(e.g., disks, bars, bulges, spiral arms, double nuclei, arcs, shells, tails, tidal debris, and accreting satellites) can be resolved by the STAGES ACS F606W images whose effective point-spread function (PSF) of $\sim 0^{\prime \prime} .1$ corresponds to $\sim 280 \mathrm{pc}$ at $z=0.165$.

\section{METHODOLOGY AND ANALYSIS}

\subsection{Galaxy Mergers in this Study}

Before outlining the methods (Sections 3.2 and 3.4) that we use to identify mergers, we briefly discuss the types of mergers that are of interest to our study, their morphological signatures, and their typical post-merger products.

According to simulations, galaxy mergers of mass ratio $M_{1} / M_{2}>1 / 10$ tend to have a significant impact on galaxy evolution. They include major mergers, which are defined to be those with a mass ratio of $1 / 4<M_{1} / M_{2} \leqslant$ $1 / 1$, as well as minor mergers with $1 / 10<M_{1} / M_{2} \leqslant 1 / 4$. Simulations show that major mergers of stellar systems typically destroy the outer disks, transforming them via violent relaxation into systems with a steep central surface brightness profile, associated with a high Sérsic index $n$ (typically $n>$ 2.5; Hopkins et al. 2009). These remnants include ellipticals and spheroids with a de Vaucouleurs-type stellar profile $(n=4)$ (e.g., Negroponte \& White 1983; Barnes \& Hernquist 1991; Mihos \& Hernquist 1996; Struck 1997; Naab \& Burkert 2001). In gas-rich major mergers, a disky component can form or survive inside the resulting spheroidal component (Hopkins et al. 2009), producing a remnant whose overall profile is less steep than a de Vaucouleurs one, with $n<4$. Irrespective of the details of the remnants, the above simulations suggest that ongoing/ recent major mergers are associated with arcs, shells, ripples, tidal tails, large tidal debris, extremely asymmetric light distributions, double nuclei inside a common body, and tidal bridges/ envelopes of light linking systems of similar mass.

Conversely, minor mergers involving a spiral galaxy and a smaller satellite of mass ratio $1 / 10<M_{1} / M_{2} \leqslant 1 / 4$, will not destroy the outer disk of the larger companion (e.g., Hernquist \& Mihos 1995; Smith 1997; Jogee et al. 1999). Typically, the smaller companion sinks via dynamical friction, may excite bars, spirals, and other non-axisymmetric perturbations in the disk of the larger galaxy, and leads to tidal heating, arcs, shells, ripples, tidal tails, tidal debris, warps, offset rings, and highly asymmetric light distributions (e.g., Quinn et al. 1993; Hernquist \& Mihos 1995; Mihos et al. 1995; Quinn et al. 1993; Smith 1997; Jogee et al. 1999; Jogee 2006 and references therein).

In this paper, we identify merging systems using two independent methods, which make use in different ways of the aforementioned morphological signatures seen in simulations. The first is a physically motivated classification system (Section 3.2), which is similar to that defined in Jogee et al. (2008, 2009), and is based on visual morphologies, stellar masses, and spectrophotometric redshifts. The second method (Section 4.2) uses the CAS merger criterion $(A>0.35$ and $A>S)$, which is based on quantitative asymmetry $(A)$, and clumpiness $(S)$ parameters derived using the CAS code (Conselice et al. 2000).

\subsection{Visual Classification of Mergers and Non-Interacting Galaxies}

We visually classify the ACS F606W images of galaxies in the sample of 893 bright $\left(R_{\text {Vega }} \leqslant 24\right)$ intermediate-mass $\left(M_{*} \geqslant\right.$ $10^{9} M_{\odot}$ ) galaxies. This sample is complete for $M_{*} \geqslant 10^{9} M_{\odot}$ (see Section 2). In Section 4.1, we will discuss whether further
Table 1

Visual Classification Results for the Sample of Bright Intermediate-Mass $\left(M_{*} \geqslant 10^{9} M_{\odot}\right)$ Galaxies

\begin{tabular}{lcc}
\hline \hline \multicolumn{1}{c}{ Visual Class } & $N_{\mathrm{VC}}$ & $f_{\mathrm{VC}}$ \\
\multicolumn{1}{c}{$(1)$} & $(2)$ & $(3)$ \\
\hline Mergers of Type 1+2a +2b & $13+3+4=20$ & $0.023 \pm 0.007$ \\
Non-interacting Irr-1 & 123 & $0.14 \pm 0.03$ \\
Non-interacting Symmetric & 743 & $0.80 \pm 0.16$ \\
Unclassifiable & 6 & $\cdots$ \\
\hline
\end{tabular}

Notes. The results of visual classification (see Section 3.2 for details) for the sample of bright intermediate-mass $\left(M_{*} \geqslant 10^{9} M_{\odot}\right)$ systems. Columns: (1) Visual class (VC); (2) $N_{\mathrm{VC}}$, the number of systems with a given VC; (3) $f_{\mathrm{VC}}$, a fraction of systems with a given $\mathrm{VC}$.

mass cuts should be applied in order to ensure the completeness of major and minor mergers, but for now we consider all the systems in the sample of 893 galaxies. A small fraction (below 1\%) of the sample could not be classified due to image defects, low signal to noise, and a highly compact appearance. As illustrated in Figure 2, the remaining galaxies are classified into five main visual classes: Mergers of Types $1,2 \mathrm{a}$, and $2 \mathrm{~b}$, and Non-interacting systems of type Symmetric and Irr-1.

The visual class "Mergers" is assigned to systems with evidence of a recent or ongoing merger of mass ratio $>1 / 10$. The mergers are subdivided into 3 groups called Type 1, Type $2 \mathrm{a}$, and Type $2 b$, because different criteria are used to identify these three types of mergers and different techniques are used to separate them into major and minor mergers. Non-interacting systems are subdivided into the visual classes of Symmetric and Irr-1. The results of the visual classification are illustrated in Tables 1-2, and Figures 3-5. Below are details of the 5 main visual classes.

(A) Mergers of Type 1. Mergers of Type 1 are systems, which appear as a single distorted remnant in the ACS F606W image (with effective PSF $\sim 0$.' 1 or $\sim 280$ pc at $z=0.165$ ), and which host morphological distortions similar to those produced in the aforementioned simulations of mergers of mass ratio $>1 / 10$. Thus, mergers of Type 1 likely represent the very advanced phases of such a merger, at a point where the two progenitor galaxies have coalesced into a single merger remnant. Among the sample of $M_{*} \geqslant 10^{9} M_{\odot}$ systems, we find 13 mergers of Type 1 . Their properties are shown in Table 2 and Figure 3 . They show morphological distortions such as tidal tails (Figure 3, Cases 10,11), shells, ripples, warps, asymmetric tidal debris and distortions (e.g., Cases 5, 6, 7, 9), or a "train-wreck" morphology (Cases 7, 10, 11, 12).

For mergers of Type 1, a single redshift and stellar mass are available. Thus, the evidence for a merger of mass ratio $>1 / 10$ does not come from a measured stellar mass ratio $M_{1} / M_{2}$, but instead is inferred from the presence of the aforementioned morphological distortions, which are seen in simulations of mergers of mass ratio $>1 / 10$.

Ideally, one would like to further separate the mergers of Type 1 into major and minor mergers, as defined in Section 3.1. However, without individual stellar masses $M_{1}$ and $M_{2}$ for the progenitors, and with only morphological distortions as a guide, this is not possible in every case since the morphological disturbances induced depend not only on the mass ratio of the progenitors, but also depend to some extent on the orbital geometry (prograde or retrograde), the gas mass fraction, and structural parameters (e.g., Mihos \& Hernquist 1996; Struck 1997; Naab \& Burkert 2001; Mihos et al. 1995; di Matteo 


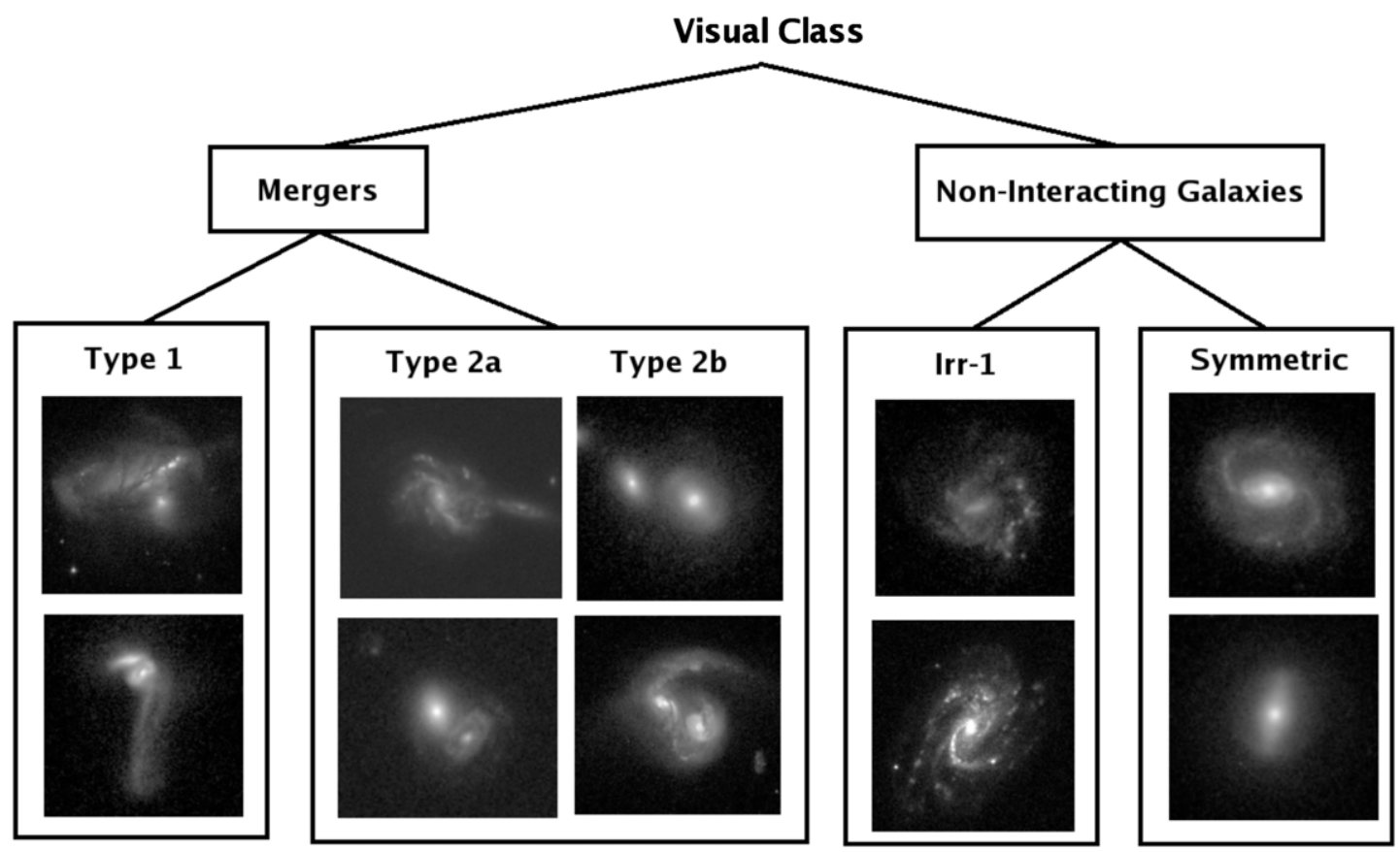

Figure 2. In our visual classification scheme (Section 3.2), systems are classified as Mergers, or Non-interacting. The mergers are sub-divided into three groups called Type 1, Type 2a, and Type 2b. Mergers of Type 1 appear, in the ACS F606W image, as a single morphologically distorted remnant, rather than two individually recognizable galaxies. The remnant hosts strong externally triggered morphological distortions similar to those seen in simulations of mergers of mass ratio $>1 / 10$, such as tidal tails, shells, ripples, warps, strongly asymmetric tidal debris, double nuclei inside a common envelope, or a "train-wreck" morphology. In contrast, mergers of Type $2 \mathrm{a}$ and $2 \mathrm{~b}$ appear in ACS images as a very close $(d<10 \mathrm{kpc})$ overlapping pair of two recognizable galaxies. Mergers of Type $2 \mathrm{~b}$ are resolved into two separate galaxies by the ground-based COMBO-17 data, while mergers of Type $2 a$ are not. Since some of these Type $2 a$ and $2 b$ systems could be due to chance line-of-sight superposition, we conservatively consider for the final analysis only those pairs where at least one member is morphologically distorted (see Table 2 and Figure 3 for details). Non-interacting systems are sub-divided into Irr-1 and Symmetric systems. Irr-1 exhibit internally triggered asymmetries, due to SF typically on scales of a few hundred parsecs. Symmetric systems include galaxies, which are relatively undistorted and are not part of the very close pairs that constitute the mergers of Types $2 \mathrm{a}$ and $2 \mathrm{~b}$.

et al. 2007). Therefore, we separate the Type 1 mergers into three groups: likely major mergers, likely minor mergers, and ambiguous cases of "major or minor" mergers as follows:

1. The class of likely major mergers includes systems, which host fairly unique tell-tale morphological distortions characteristic of a major merger, such as a train-wreck morphology (e.g., Cases 7, 10, 11, 12 in Figure 3), or two nuclei of similar luminosities.

2. A system is classified as a likely minor merger if the outer disk has survived a recent merger. We base this on the results of simulations of mergers involving spiral galaxies where the outer disk of the spiral survives a minor merger, but not a major merger (Section 3.1). This is not true in every case: major mergers of extremely gas-rich disks with low SF efficiency can lead to a remnant with an extended stellar disk (Robertson et al. 2004), but such mergers are unlikely to be relevant for our study, which focuses on intermediatemass $\left(M_{*} \geqslant 10^{9} M_{\odot}\right)$ systems at redshifts well below 1 . An additional secondary criterion for classifying a system as a likely minor merger is that the light from morphological distortions, tidal debris, or accreted system in the surviving disk is a small fraction (between $1 / 4$ and 1/10) of the total luminosity of the disk. Examples include Cases 8, 9, 13 in Figure 3 and Table 2.

3. The class of ambiguous "major or minor" merger is assigned to systems hosting distortions, which could be due to both a major and a minor merger. Examples are Cases $1-6$ in Figure 3 and Table 2.

(B) Mergers of Types $2 a$ and $2 b$. Mergers of Types $2 \mathrm{a}$ and $2 \mathrm{~b}$ are systems, which appear in ACS images as a very close (separation $d<10 \mathrm{kpc}$ ) overlapping pair of two galaxies, and whose properties suggest they are in the very late phases of a merger of mass ratio above $1 / 10$. The difference between mergers of Type $2 \mathrm{a}$ and $2 \mathrm{~b}$ is that the latter are resolved into two separate galaxies by the ground-based COMBO-17 data of resolution $\sim 1^{\prime \prime} .5$ (corresponding to $\sim 4.3 \mathrm{kpc}$ at $z \sim 0.165$ ), while the former are not. Thus, mergers of Type $2 \mathrm{a}$ only have a single redshift and a single stellar mass for the pair, while mergers of Type $2 \mathrm{~b}$ have stellar masses $\left(M_{1}, M_{2}\right)$ and spectrophotometric redshifts $\left(z_{1}, z_{2}\right)$ for both pair members.

We focus only on very close pairs (with $d<10 \mathrm{kpc}$ ), and ignore widely separated pairs for several reasons. First, in this work, we are interested in systems with evidence for a recent or ongoing merger of mass ratio $>1 / 10$. The very close pairs represent the very late phases of such a merger, such that gravitationally bound members can coalesce into a remnant on a short timescale, comparable to the visibility timescale over which the morphological distortions in mergers of Type 1 persist. Second, we expect the morphological distortions from the ongoing merger to be more prominent in close pairs than widely separated ones. These morphological signatures in turn make it easier to separate real pairs from projection pairs. Third, as shown by our Monte Carlo simulations (see below), the statistical contamination from projection pairs increases significantly for distant pairs.

When identifying potential mergers of Type $2 b$, we consider very close pairs where one pair member has $M_{*} \geqslant 10^{9} M_{\odot}$, and its companion satisfies the following two conditions. First, the companion has a mass such that the mass ratio is in the range $1 / 10<M_{1} / M_{2} \leqslant 1 / 1$. Note that this condition allows for pairs where the companion can be of higher, as well as lower mass (see 

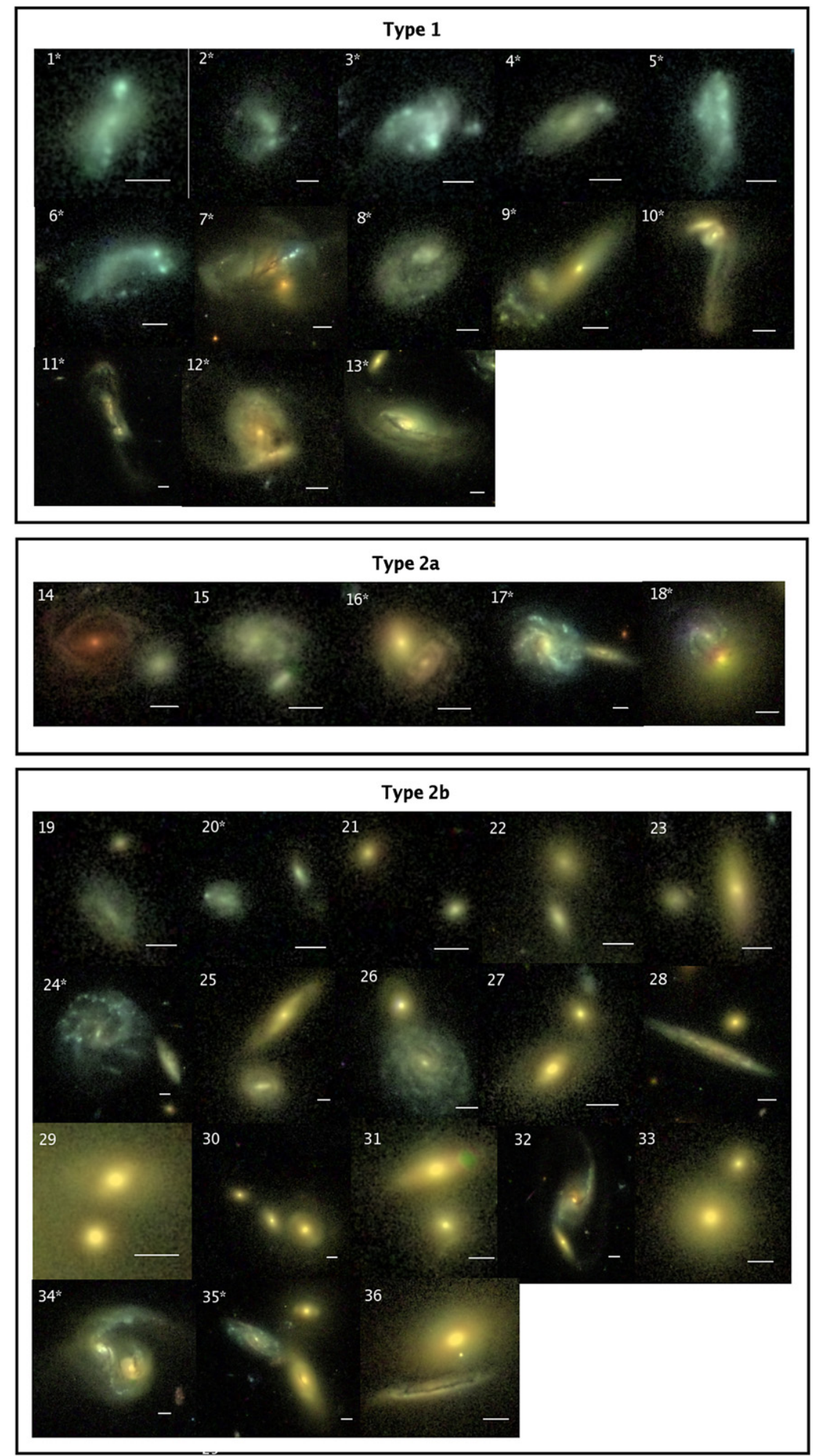

Figure 3. Top, middle, and lower panels show the ACS images of systems visually classified as mergers of Type 1 and potential mergers of Type $2 \mathrm{a}$ and $2 \mathrm{~b}$, respectively (see Section 3.2 for details). White horizontal bars denote a scale of $1^{\prime \prime}$ or $2.8 \mathrm{kpc}$ at $z \sim 0.165$. The properties of the mergers are listed in Table 2. Top panel: the 13 distorted remnants classified as Type 1 mergers hosts strong externally triggered morphological distortions similar to those seen in simulations of mergers of mass ratio $>1 / 10$, such as tidal tails (e.g., Cases 10,11), shells, ripples, warps, asymmetric tidal debris, and distortions (e.g., Cases 5,6,8,9), double nuclei inside a common envelope, or a "train-wreck" morphology (Cases 7, 10,11,12). The Type 1 mergers can be divided into major mergers $($ Cases $7,10,11,12)$, minor mergers $($ Cases $8,9,13$ ), and ambiguous "major or minor" mergers (Cases 1-6) as described in Section 3.2. Middle and lower panels: the 23 potential mergers of Type 2a and 2b shown here include both real and projection very close $(d<10 \mathrm{kpc})$ pairs. The 7 likely real pairs (Cases 16-18, 20, 24, 34, 35) are denoted with a starred identification number and contain at least one galaxy with morphological distortions indicative of a galaxy-galaxy interaction. In the final analysis, only 13 distorted mergers of Type 1 and 7 distorted mergers of Types $2 \mathrm{a}$ and $2 \mathrm{~b}$ are used. Type $2 \mathrm{~b}$ pairs are resolved by COMBO-17 data into two galaxies with separate redshifts and stellar masses, and their stellar mass ratio (listed in Table 2) is used to divide Type $2 \mathrm{~b}$ mergers into major mergers (Cases 20-22, 24-26, 28, 29, 23, 34) and minor mergers (Cases $19,23,27,30,31,33,35,36$ ). For the Type 2a pairs, which are unresolved by COMBO-17, the ACS-based luminosity ratio (listed in Table 2) of the pair members is used to divide them into major (Cases 16, 18) and minor (Cases 14, 15, 17) mergers.

(A color version of this figure is available in the online journal.) 
Table 2

Visual Classification of Mergers in the Sample of Bright Intermediate-Mass $\left(M_{*} \geqslant 10^{9} M_{\odot}\right)$ Galaxies

\begin{tabular}{|c|c|c|c|c|c|c|}
\hline $\begin{array}{c}\text { Merger ID } \\
\text { (1) } \\
\end{array}$ & $\begin{array}{c}\text { Merger Mass } \\
\qquad\left(10^{9} M_{\odot}\right) \\
(2)\end{array}$ & $\begin{array}{c}M_{1} \\
\text { for Type } 2 b \\
\left(10^{9} M_{\odot}\right) \\
(3)\end{array}$ & $\begin{array}{c}M_{2} \\
\text { for Type } 2 b \\
\left(10^{9} M_{\odot}\right) \\
(4)\end{array}$ & $\begin{array}{c}M_{1} / M_{2} \\
\text { for Type } 2 b \\
(5)\end{array}$ & $\begin{array}{c}L_{1} / L_{2} \\
\text { for Type } 2 \mathrm{a} \\
(6)\end{array}$ & $\begin{array}{c}\text { Major, Minor, } \\
\text { or Ambiguous } \\
\text { (7) }\end{array}$ \\
\hline \multicolumn{7}{|c|}{ Mergers of Type 1} \\
\hline $1^{\star}$ & 1.22 & & & & & Ambiguous \\
\hline $2^{\star}$ & 1.31 & & & & & Ambiguous \\
\hline $3^{\star}$ & 1.62 & & & & & Ambiguous \\
\hline $4^{\star}$ & 1.64 & & & & & Ambiguous \\
\hline $5^{\star}$ & 1.64 & & & & & Ambiguous \\
\hline $6^{\star}$ & 2.73 & & & & & Ambiguous \\
\hline $7^{\star}$ & 6.66 & & & & & Major \\
\hline $8^{\star}$ & 8.06 & & & & & Minor \\
\hline $9^{\star}$ & 23.3 & & & & & Minor \\
\hline $10^{\star}$ & 24.4 & & & & & Major \\
\hline $11^{\star}$ & 46.0 & & & & & Major \\
\hline $12^{\star}$ & 122.1 & & & & & Major \\
\hline $13^{\star}$ & 191.5 & & & & & Minor \\
\hline \multicolumn{7}{|c|}{ Potential Mergers of Type $2 \mathrm{a}$} \\
\hline 14 & 2.32 & & & & 6.9 & Minor \\
\hline 15 & 5.25 & & & & 6.0 & Minor \\
\hline $16^{\star}$ & 13.0 & & & & 1.4 & Major \\
\hline $17^{\star}$ & 36.5 & & & & 5.0 & Minor \\
\hline $18^{\star}$ & 281.5 & & & & 1.1 & Major \\
\hline \multicolumn{7}{|c|}{ Potential Mergers of Type $2 b$} \\
\hline 19 & 1.52 & 1.32 & 0.20 & 6.60 & & Minor \\
\hline $20^{\star}$ & 1.95 & 1.12 & 0.83 & 1.35 & & Major \\
\hline 21 & 3.24 & 2.48 & 0.76 & 3.26 & & Major \\
\hline 22 & 7.90 & 5.34 & 2.56 & 2.09 & & Major \\
\hline 23 & 14.6 & 13.3 & 1.33 & 10. & & Minor \\
\hline $24^{\star}$ & 20.0 & 15.1 & 4.94 & 3.06 & & Major \\
\hline 25 & 21.0 & 14.3 & 6.73 & 2.12 & & Major \\
\hline 26 & 33.6 & 24.1 & 9.45 & 2.55 & & Major \\
\hline 27 & 43.5 & 35.6 & 7.86 & 4.53 & & Minor \\
\hline 28 & 44.3 & 26.1 & 18.2 & 1.43 & & Major \\
\hline 29 & 58.9 & 37.4 & 21.5 & 1.74 & & Major \\
\hline 30 & 60.5 & 52.2 & 8.34 & 6.26 & & Minor \\
\hline 31 & 79.2 & 71.8 & 7.38 & 9.73 & & Minor \\
\hline 32 & 110.6 & 83.1 & 27.5 & 3.02 & & Major \\
\hline 33 & 126.4 & 113.8 & 12.6 & 9.03 & & Minor \\
\hline $34^{\star}$ & 154.6 & 117.0 & 37.6 & 3.11 & & Major \\
\hline $35^{\star}$ & 202.6 & 169.0 & 16.6 & 10.18 & & Minor \\
\hline 36 & 238.6 & 214.6 & 24.0 & 8.94 & & Minor \\
\hline
\end{tabular}

Notes. The table shows the visually classified mergers, which are identified in the sample of 893 bright $\left(R_{\text {Vega }} \leqslant 24\right)$ intermediate-mass $\left(M_{*} \geqslant 10^{9} M_{\odot}\right)$ galaxies. The mergers are sub-divided into three groups: mergers of Type 1 and potential mergers of types $2 \mathrm{a}$ and $2 \mathrm{~b}$, according to the criteria outlined in Section 3.2. Columns: (1) numerical identifier corresponding to the potential mergers in Figure 3. Note that the very close $(d<10 \mathrm{kpc})$ pairs listed as potential mergers of Types $2 \mathrm{a}$ and $2 \mathrm{~b}$ in this table includes both real and projection pairs. The likely real pairs are marked with a star in Column 1 and contain at least one galaxy with morphological distortions indicative of a galaxy-galaxy interaction. In the final analysis, only the 13 distorted mergers of Type 1 and the 7 distorted mergers of Types $2 \mathrm{a}$ and $2 \mathrm{~b}$ are used; (2) the mass of the merger. For pairs of Type $2 \mathrm{~b}$, which are resolved into two galaxies by COMBO- 17 data, the mass cited is the sum $\left(M_{1}+M_{2}\right)$ of the pair members; (3) for mergers of Type $2 \mathrm{~b}$, the mass $M_{1}$ of one galaxy in the pair; (4) for mergers of Type $2 \mathrm{~b}$, the mass $M_{2}$ of the second galaxy in the pair; (5) for mergers of Type $2 \mathrm{~b}$, the mass ratio $\left(M_{1} / M_{2}\right)$; (6) for mergers of Type 2a, the luminosity ratio $\left(L_{1} / L_{2}\right)$, measured from ACS images; (7) the classification of the merger into major merger, minor merger, or ambiguous "major or minor," according to the criteria outlined in Section 3.2.

Table 2). Second, the companion has similar spectrophotometric redshift, such that the absolute value of $\left(z_{1}-z_{2}\right)$ is less than or equal to the $1 \sigma$ redshift error $\sigma_{z}$. The latter criterion helps to remove some of the false projection pairs. However, there is still residual contamination by false pairs as $\sigma_{z}$ is fairly large (see Section 2; $\sigma_{z} \sim 0.028$ at $R_{\text {Vega }}=23.0$ ).

We identified 5 and 18 potential candidate mergers of Types $2 \mathrm{a}$ and $2 \mathrm{~b}$, respectively, in our final sample of 886 systems. They are shown in Figure 3 and Table 2. We use the term "po- tential mergers of Types $2 \mathrm{a}$ and $2 \mathrm{~b}$ " when describing the 23 very close $(d<10 \mathrm{kpc})$ galaxy pairs listed in Table 2 (Cases 14-36), because these pairs could be a mix of real pairs and false projection pairs caused by chance line-of-sight superposition. In order to minimize the contamination from false projection pairs, we only include in our final analysis those pairs where at least one galaxy shows morphological distortions indicative of a galaxy-galaxy interaction. Such pairs are most likely to be real pairs, as opposed to projection pairs, and we will use 


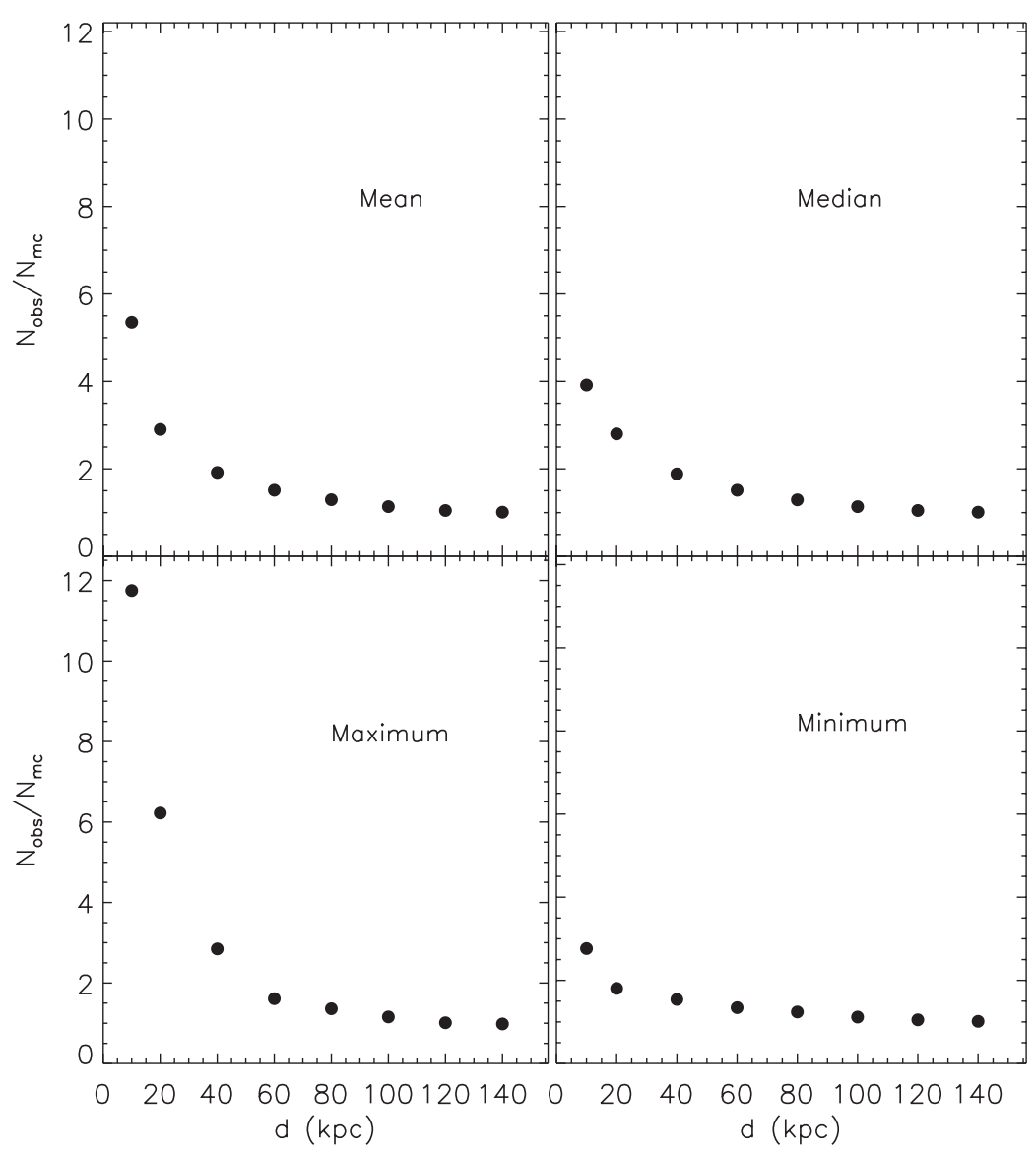

Figure 4. Ratio $\left(N_{\mathrm{obs}} / N_{\mathrm{mc}}\right)$ for pairs of different projected separations $d$, where $N_{\mathrm{obs}}$ and $N_{\mathrm{mc}}$ are the numbers of pairs measured in the observations and random pairs from the Monte Carlo simulations, respectively. See Section 3.2 for details. The four panels represent the mean, median, minimum, and maximum distribution as labeled. At smaller separations of $d<50 \mathrm{kpc},\left(N_{\mathrm{obs}} / N_{\mathrm{mc}}\right)$ is above 1 , suggesting that random chance superposition cannot fully account for all the observed pairs.

these systems to set a firm lower limit to the merger fraction in Section 4.1. We find 7 such pairs (Cases 16-18, 20, 24, $34,35)$, which are marked with a star in Column 1 of Table 2. This suggests that the remaining $16 / 23$ or up to $70 \%$ of the Types $2 \mathrm{a}$ and $2 \mathrm{~b}$ could potentially be pairs in projection, due to the dense cluster environment.

As a second step to gauge the level of contamination from projection pairs, we performed a Monte Carlo simulation. We generated a random galaxy distribution which has an identical redshift distribution to that of our sample of intermediate-mass $\left(M_{*} \geqslant 10^{9} M_{\odot}\right)$ galaxies, over the STAGES $0.5 \times 0.5$ field. We ran 100 realizations of this Monte Carlo simulation. For each realization, we took the ratio $\left(N_{\mathrm{obs}} / N_{\mathrm{mc}}\right)$ of observed to simulated Monte Carlo pairs at different separations $(d)$ out to $140 \mathrm{kpc}$. The same criteria outlined in Section 3.2 to identify the pairs in the observations are applied to the simulated galaxies. Namely, we apply the condition that the absolute value of the difference in spectrophotometric redshifts of the galaxies in a pair must be less than or equal to the $1 \sigma$ redshift error $\sigma_{z}$, in order for the galaxies to be counted as a pair. Figure 4 shows the ratio $\left(N_{\mathrm{obs}} / N_{\mathrm{mc}}\right)$ plotted as a function of $d$, with the four panels showing the mean, median, minimum, and maximum values of $\left(N_{\mathrm{obs}} / N_{\mathrm{mc}}\right)$. Our results are qualitatively similar to those found by Kartaltepe et al. (2007). At large separations of $d>50 \mathrm{kpc},\left(N_{\mathrm{obs}} / N_{\mathrm{mc}}\right)$ is below 1 , and false projection pairs can dominate the statistics. At small separations of $d<20 \mathrm{kpc}$, $\left(N_{\text {obs }} / N_{\text {mc }}\right)$ is well above 1 , suggesting that random chance superposition cannot fully account for all the observed pairs.
Thus, by picking pairs with a separation of $d<20 \mathrm{kpc}$, one can reduce the contamination from false pairs. For $d<20 \mathrm{kpc}$, the mean value of the contamination level (defined as the fraction of observed pairs that are false) ranges from $16 \%$ to $55 \%$ and has a median value of $36 \%$. The maximum contamination level is a factor of $\sim 1.3$ lower than the factor of $70 \%$, which we derived in the preceding paragraph, by assuming that all pairs without morphological distortions are projection pairs. One possible explanation for the difference is that the afore-described Monte Carlo simulations do not take into account the large-scale structure and possible overdensity of galaxies associated with the central parts of the supercluster.

We next attempt to separate the mergers of Type $2 \mathrm{a}$ and $2 \mathrm{~b}$ into major and minor mergers. For mergers of Type $2 b$, where stellar masses $\left(M_{1}, M_{2}\right)$ are available for each pair member, we can use the stellar mass ratio $M_{1} / M_{2}$ (see Table 2). By definition, systems with $1 / 10<M_{1} / M_{2} \leqslant 1 / 4$ and $1 / 4<M_{1} / M_{2} \leqslant 1$ are classified, respectively, as minor and major mergers. As shown in Table 2, potential major mergers of Type $2 \mathrm{~b}$ are Cases 20-22, 24-26, 28, 29, 32, and 34, while Cases 19, 23, 27, 30, 31, 33, 35 , and 36 are potential minor mergers of Type $2 b$.

Mergers of Type 2a, however, only have a single redshift and a single stellar mass, and hence the stellar mass ratio of the pair is not directly measurable. Instead, we use the stellar light ratio $L_{1} / L_{2}$, measured from the ACS images, as an approximate proxy (see Table 2). Examples of likely major mergers of Type $2 \mathrm{a}$ are Cases 16 and 18 in Figure 3, while Cases 14, 15, and 17 are examples of likely minor mergers of Type $2 \mathrm{a}$. 

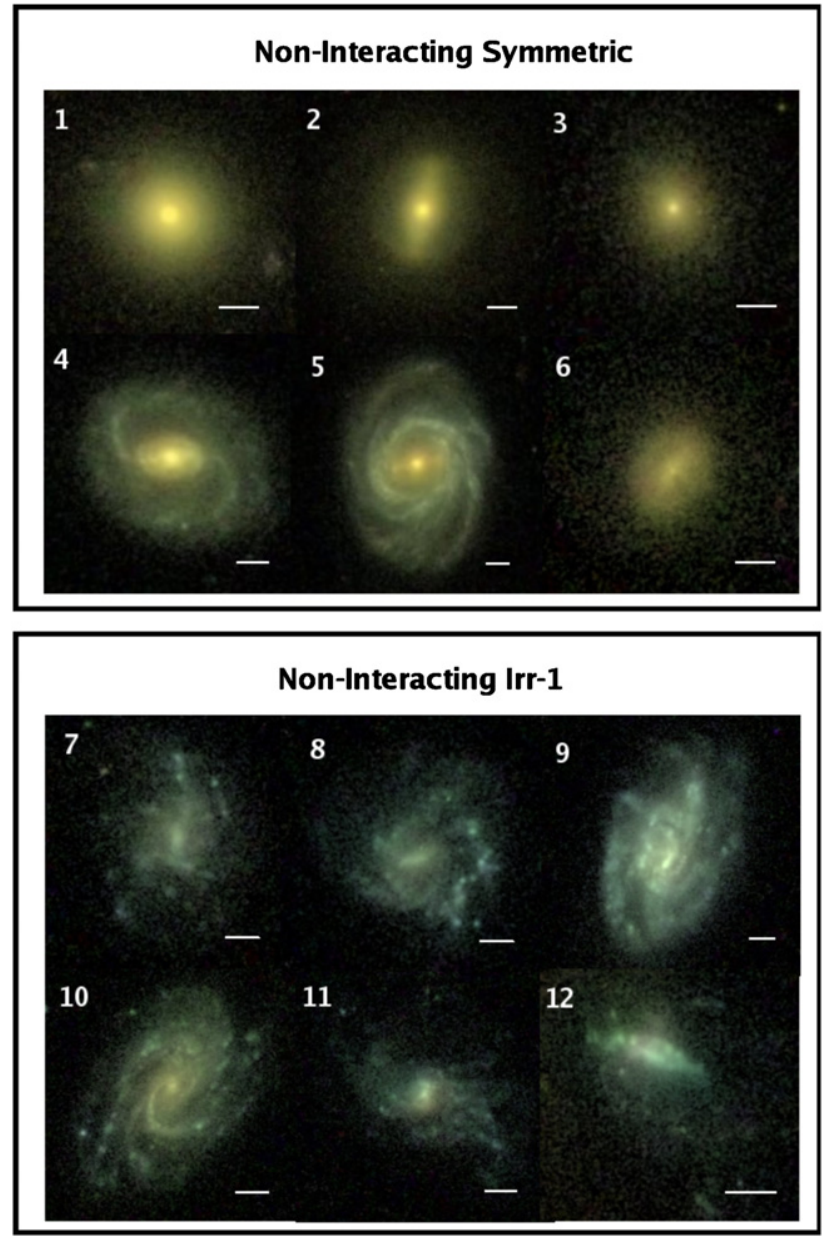

Figure 5. ACS F606W images of some of the systems visually classified as Noninteracting Symmetric (Cases 1-6) and Non-interacting Irr-1 (Cases 7-12). See Section 3.2 for details.

(A color version of this figure is available in the online journal.)

(C) Non-interacting systems of Type Symmetric and Irr-1. The remaining systems, which show no indication of a recent merger, according to the criteria outlined in Sections A and $\mathrm{B}$ above, are classified as non-interacting systems. They are subdivided into two groups called "Non-interacting Irr-1" and "Non-interacting Symmetric." "Non-interacting Irr-1" systems exhibit internally triggered asymmetries, typically on scales of a few hundred parsecs. These asymmetries are generally due to stochastic SF and/or low ratios of rotational to random velocities. They can impart a clumpy morphology to a galaxy and are different from externally driven distortions, such as tails and ripples, which are often correlated on scales of several kpc. The class of "Non-Interacting Symmetric" consists of galaxies, which are relatively undistorted. Note that this class includes the undistorted galaxies, which are part of these Type 2a and $2 \mathrm{~b}$ pairs identified as projection pairs in Section B. Examples of "Non-interacting Irr-1" and "Non-interacting Symmetric" galaxies are shown in Figure 5.

In summary after performing the visual classification of the 893 bright $\left(R_{\text {Vega }} \leqslant 24\right)$ intermediate mass $\left(M_{*} \geqslant 10^{9}\right)$ galaxies, we find the following results. We identify 13 merger remnants of Type 1 (Cases 1-13 in Table 2 and Figure 3), and 7 close pairs of Types $2 \mathrm{a}$ and $2 \mathrm{~b}$ (Cases 16-18, 20, 24, 34, 35 in Table 2 and Figure 3) where at least one galaxy in the close pair shows morphological distortions indicative of a galaxy- galaxy interaction. Such pairs are most likely to be real pairs, as opposed to projection pairs. We discount those very close pairs where none of the members are morphologically distorted. Our final sample includes 20 mergers and 866 non-interacting galaxies (123 Irr-1 + 743 Symmetric; see Table 1).

\subsection{Tests on the Visual Classes}

Several tests were performed on the visual classes described in $\mathrm{A}, \mathrm{B}$, and $\mathrm{C}$ in Section 3.2. The visual classifications were calibrated by having three classifiers $(\mathrm{AH}, \mathrm{IM}, \mathrm{SJ})$ train on a set of $\sim 100$ nearby field galaxies. The full supercluster sample was then classified by $\mathrm{AH}$ and IM, with all ambiguous cases and interacting galaxies discussed by all three classifiers for final determination. Random checks on sub-samples of galaxies were performed by SJ. We denote as $f_{\mathrm{VC}}$ the fraction of intermediatemass $\left(M_{*} \geqslant 10^{9} M_{\odot}\right)$ systems with different visual classes (e.g., mergers of Types $1,2 \mathrm{a}$, and $2 \mathrm{~b}$, and non-interacting systems of type Symmetric and Irr-1). In order to gauge the subjectivity inherent in any visual classification scheme, we estimate the maximum difference in the value of $f_{\mathrm{VC}}$ across the classifiers $\left(\delta f_{\mathrm{VC}}\right)$. The maximum percentage difference $P$ defined as $\left(100 \times \delta f_{\mathrm{VC}} / f_{\mathrm{VC}}\right)$ is $4 \%, 19 \%$, and $16 \%$ respectively for the visual classes of Non-interacting Symmetric, Non-interacting Irr-1, and Mergers. We conservatively adopt a value of $20 \%$ for $P$ on the mean merger fraction, as a measure of the inherent subjectivity in the visual classification. When citing the final error bar on $f_{\mathrm{VC}}$, we combine in quadrature the term $\delta f_{\mathrm{VC}}$, which represents the subjectivity across classifiers, and the binomial error term $\left(\sqrt{f_{\mathrm{VC}}\left(1-f_{\mathrm{VC}}\right) / N}\right)$, where $N$ is the sample size of 886 systems. The raw values for $f_{\mathrm{VC}}$ are shown in Table 1.

It is also interesting to explore any systematic effects affecting the mergers we identified visually. The criteria we used, based on morphological distortions, might beg the question of whether these mergers are biased toward a gas-rich population. The extent to which a galaxy develops morphological distortions in an interaction depends on whether the encounter is prograde or retrograde, the interaction phase, the gas content, and the mass ratio $\left(M_{1} / M_{2}\right)$. Di Matteo et al. (2007) found that the most influential parameters are the mass ratio and orbital geometry, and not the gas content alone. The visibility timescale $\left(t_{\mathrm{vis}}\right)$ on which the morphological signatures persist also depend on the orbital geometry, gas content, and the mass ratio. We do not have a direct measurement of the gas content of galaxies in A901/902, so we attempt an indirect first-order test in order to verify whether our methodology preferentially picks gas-rich mergers. We visually classify sample galaxies into three broad classes: "highly smooth," "highly clumpy," and "other." If a system shows a high degree of clumpiness (i.e., very patchy regions that are likely associated with significant amounts of gas, dust, and SF), it is classified as "highly clumpy." Conversely, systems with a very smooth non-clumpy appearance are classified as "highly smooth."

Out of the 20 mergers and 866 non-interacting galaxies, we find that $6 / 20(30 \%)$ and $210 / 866(24 \%)$ are classified as "highly clumpy," respectively. The comparable fraction of "highly clumpy" systems among non-interacting systems and mergers suggests that our method for identifying mergers does not show a strong bias toward preferentially selecting mergers, which are highly clumpy, gas-rich, and star forming. It is also interesting to recall here that our visual classification scheme takes special care to distinguish between Noninteracting Irr-1 and mergers (Section 3.2). The Non-interacting Irr-1 galaxies were defined as those where internally trig- 
gered small-scale asymmetries, often due to SF, are present. Thus, as expected, if we separate our non-interacting galaxies into Irr-1 and Symmetric, we find that a large fraction of the Non-interacting Irr-1 (111/123 or 90\%) are "highly clumpy," compared to $99 / 713$ (14\%) of the Non-interacting Symmetric systems.

\subsection{CAS: Quantitative Method for Capturing Interacting Galaxies}

Using the CAS code (Conselice et al. 2000; Conselice 2003), quantitative structural parameters measuring the concentration $(C)$, asymmetry $(A)$, and clumpiness $(S)$ were derived for the supercluster galaxies. CAS was run on the F606W images and the segmentation maps produced during the original source extraction (Caldwell et al. 2008) were used to mask neighboring galaxies.

The CAS concentration index $C$ (Bershady et al. 2000) is proportional to the logarithm of the ratio of the $80 \%-20 \%$ curve of growth radii within 1.5 times the Petrosian inverted radius at $r(\eta=0.2)$ :

$$
C=5 \times \log \left(\frac{r_{80 \%}}{r_{20 \%}}\right) .
$$

The CAS code derives the asymmetry index $A$ (Conselice 2003 ) by rotating a galaxy image by $180^{\circ}$, subtracting the rotated image from the original image, summing the absolute intensities of the residuals, and normalizing the sum to the original galaxy flux. CAS improves the initial input center with the IRAF task "imcenter" and then performs a further refinement within a $3 \times 3$ grid, picking the center that minimizes $A$.

The clumpiness parameter $S$ is a quantitative measure of the high spatial frequency patchiness of a galaxy. It is defined as the summation of the difference of the galaxy's original flux and the flux of an image with the high frequency structures muted by smoothing over a filter on order of the clumpiness, taking into account the background flux over that same area. This measurement is then divided by the summation of the original flux of the galaxy to obtain the clumpiness parameter $S$.

Conselice et al. (2000) argue that the CAS merger criterion $(A>S$ and $A>0.35)$ at $\lambda_{\text {rest }}>4500 \AA$ can be used to capture galaxies which have strong asymmetries indicative of major mergers. However, calibrations of the asymmetry value with $N$-body simulations (Conselice 2006; Lotz et al. 2008) have shown that for galaxies involved in major mergers of mass ratios $1: 1-1: 3$, the asymmetries vary as a function of time during the merger. The $A$ value reaches a peak near the midpoint of the merger and falls off both before and after this time to less than 0.35 . The criterion $A>0.35$ is fulfilled only $\sim 1 / 3$ of the time for major mergers in these simulations, while minor mergers of mass ratios below 1:5 have $A$ values significantly lower than 0.35 .

Furthermore, in intermediate-mass $\left(M_{*} \geqslant 10^{9} M_{\odot}\right)$ field galaxies at $z \sim 0.24-0.80$, recent work (Jogee et al. 2008, 2009; Miller et al. 2008) has shown that the CAS criterion typically only picks up 50\%-70\% of galaxies visually typed as being disturbed or interacting. These results caution against using CAS without complementary visual classifications in field galaxies.

The effectiveness of the CAS criterion has not been explored in cluster environments where the dominant galaxy population is more gas-poor than in the field. In Section 4.2, we present the merger fraction $\left(f_{\mathrm{CAS}}\right)$ from CAS and perform one of the first systematic comparisons to date between CAS-based and visual classification results in clusters.

\section{RESULTS AND DISCUSSION}

\subsection{Merger Fraction in A901/902 from Visual Classification}

Next we discuss how to define and estimate the merger fraction. Our goal is to estimate the fraction of $f_{\text {merge }}$ of systems with stellar mass above an appropriately chosen mass cut $M_{\text {cut }}$, which are involved in mergers of mass ratio $\geqslant 1 / 10$. The merger fraction $f_{\text {merge }}$ is computed as $\left(N_{\text {merge }} / N_{\text {tot }}\right)$, where $N_{\text {merge }}$ is the number of major and minor mergers involving galaxies with $M_{*} \geqslant M_{\text {cut }}$, and $N_{\text {tot }}$ is the total number of galaxies with $M_{*} \geqslant M_{\text {cut }}$.

It is important to determine the minimum stellar masses of the major and minor mergers involving galaxies with $M_{*} \geqslant M_{\text {cut }}$ and thereby assess for what value of $M_{\text {cut }}$ we can trace such mergers. Major merger pairs (defined as having mass ratio $1 / 4<M_{1} / M_{2} \leqslant 1$ ) of mass ratio $1: 1$ to $1: 3$ will have minimum stellar masses ranging from $2 \times M_{\text {cut }}$ to $4 \times M_{\text {cut }}$, in cases where galaxies of mass $M_{*} \geqslant M_{\text {cut }}$ merge with systems at least as massive as themselves. However, if galaxies of mass $M_{*} \geqslant M_{\text {cut }}$ merge with lower mass systems, the minimum mass of 1:1 to 1:3 mergers will range from $2 \times M_{\text {cut }}$ to $\left(4 \times M_{\text {cut }} / 3\right)$. Taken together, the above constraints imply that we are complete for major mergers involving galaxies with $M_{*} \geqslant M_{\text {cut }}$, as long as we can trace 1:3 major mergers with a minimum stellar mass of $\left(4 \times M_{\text {cut }} / 3\right)$.

Repeating the exercise for minor mergers (defined as having $\left.1 / 10<M_{1} / M_{2} \leqslant 1 / 4\right)$, it follows that the minimum stellar mass for 1:4 to $1: 9$ mergers ranges from $5 \times M_{\text {cut }}$ to $9 \times M_{\text {cut }}$ or from $\left(5 \times M_{\text {cut }} / 4\right)$ to $\left(10 \times M_{\text {cut }} / 9\right)$, depending on whether galaxies of mass $M_{*} \geqslant M_{\text {cut }}$ merge with systems of higher mass or lower mass. Thus, we are complete for minor mergers involving galaxies with $M_{*} \geqslant M_{\text {cut }}$, as long as we can trace 1:9 minor mergers with a minimum stellar mass of $\left(10 \times M_{\text {cut }} / 9\right)$

For what value of $M_{\text {cut }}$ are these conditions satisfied by the mergers of Types $1,2 \mathrm{a}$, and $2 \mathrm{~b}$, which we visually identified in the final sample (Section 3.2)? Since our final sample is complete for $M_{*} \geqslant 10^{9} M_{\odot}$, it follows that we can identify all mergers of Types 1 and $2 \mathrm{a}$ with $M_{*} \geqslant 10^{9} M_{\odot}$ from this sample. If we impose this value to the afore-defined criteria of $\left(10 \times M_{\text {cut }} / 9\right)$ and $\left(4 \times M_{\text {cut }} / 3\right)$, it implies that a mass cut $M_{\text {cut }} \geqslant 0.9 \times 10^{9} M_{\odot}$ would allow us to be complete for major and minor mergers of Types 1 and $2 \mathrm{a}$. However, the situation is different for the mergers of Type $2 b$ (close pairs resolved into two galaxies by COMBO-17) because the individual galaxies making up the pair are complete only for $M_{*} \geqslant 10^{9} M_{\odot}$. As a result, we can only completely trace 1:3 major mergers of Type $2 b$ with total mass $\geqslant 4.0 \times 10^{9} M_{\odot}$. If we impose this value to the aforedefined criterion of $\left(4 \times M_{\text {cut }} / 3\right)$, it implies that a mass cut of $M_{\text {cut }} \geqslant 3.0 \times 10^{9} M_{\odot}$ is needed to ensure completeness for major mergers of Type $2 b$. Similarly, we can only completely trace 1:9 minor mergers of Type $2 b$ with total mass $\geqslant 9.0 \times 10^{9} M_{\odot}$, which implies that a mass cut $M_{\text {cut }} \geqslant 9.0 \times 10^{9} M_{\odot}$ is needed to completely trace all minor mergers of Type $2 \mathrm{~b}$.

Using a mass cut $M_{\text {cut }} \geqslant 9.0 \times 10^{9} M_{\odot}$ for computing the merger fraction $f_{\text {merge }}$ would allow us to be complete for major and minor mergers of Types $1,2 \mathrm{a}$, and $2 \mathrm{~b}$. However, it leads to very small number statistics and is not viable. We therefore explore the value of $f_{\text {merge }}$ for the less severe cut of $M_{\text {cut }} \geqslant 3.0 \times 10^{9} M_{\odot}$, as well as for $M_{\text {cut }} \geqslant 10^{9} M_{\odot}$. The cut of $M_{\text {cut }} \geqslant 3.0 \times 10^{9} M_{\odot}$ ensures that we are complete for major and minor mergers of Types $1,2 \mathrm{a}$, but leaves us incomplete for mergers of Type $2 b$. It is encouraging that both cuts yield 
Table 3

Merger Fraction for Different Mass Cuts

\begin{tabular}{|c|c|c|c|c|c|c|c|c|c|}
\hline $\begin{array}{c}M_{\text {cut }} \\
\left(M_{\odot}\right) \\
(1)\end{array}$ & $\begin{array}{l}N_{\text {tot }} \\
(2) \\
\end{array}$ & $N_{1}$ & $N_{2 \mathrm{a}}($ all $)$ & $N_{2 \mathrm{~b}}($ all $)$ & $N_{\text {merge }}$ (all) & $f_{\text {merge }}$ (all) & $f_{\text {major }}($ all $)$ & $f_{\text {minor }}($ all $)$ & $\begin{array}{c}f_{\text {ambig }}(\text { all }) \\
(10)\end{array}$ \\
\hline $1 \times 10^{9}$ & 886 & 13 & $3(5)$ & $4(18)$ & $20(36)$ & $0.023 \pm 0.007(0.041 \pm 0.01)$ & $9 / 886(16 / 886)$ & $5 / 886(14 / 886)$ & $6 / 886(6 / 886)$ \\
\hline $3 \times 10^{9}$ & 609 & 7 & $3(4)$ & $3(16)$ & $13(27)$ & $0.021 \pm 0.007(0.033 \pm 0.01)$ & $8 / 609(15 / 609)$ & $3 / 609(12 / 609)$ & $0(0)$ \\
\hline
\end{tabular}

Notes. The merger fraction $f_{\text {merge }}$ among systems above a mass cut $\left(M_{\text {cut }}\right)$ is computed as $\left(N_{\text {merge }} / N_{\text {tot }}\right)$, where $N_{\text {merge }}$ is the number of galaxies with $M_{*} \geqslant M_{\text {cut }}$ involved in major and minor mergers, and $N_{\text {tot }}$ is the total number of systems with $M_{*} \geqslant M_{\text {cut }}$. This table shows the value of $f_{\text {merge }}$ for different mass cuts. Columns: (1) mass cut $M_{\text {cut }}$ in units of $M_{\odot}$; (2) the total number $N_{\text {tot }}$ of systems with $M_{*} \geqslant M_{\text {cut }}$; (3) number $N_{1}$ of mergers of Type 1 for this mass cut; (4) number $N_{2 \mathrm{a}}$ of mergers of Type 2a for this mass cut.; (5) number $N_{2 \mathrm{~b}}$ of mergers of Type 2b for this mass cut; (6) $N_{\text {merge }}$, the total number of mergers of Types 1, 2a, and 2b for this mass cut; (7) the merger fraction $f_{\text {merge }}$, computed as ( $\left.N_{\text {merge }} / N_{\text {tot }}\right)$; (8) the major merger fraction, based on the classification in Column 8 of Table 2; (9) same as (8), but for the minor merger fraction; (10) same as (8), but for the fraction of ambiguous mergers, which could be either major or minor mergers. In Columns 4-10, the first number without brackets apply to the case we only consider the 13 Type 1 mergers and the subset of 7 reliable Type 2a and 2b mergers, which are likely to be real pairs as they contain at least one member galaxy with morphological distortions indicative of a galaxy-galaxy interaction. The value in bracket applies to the case where we include the 13 Type 1 mergers and all the 23 mergers of Types $2 \mathrm{a}$ and $2 \mathrm{~b}$ listed in Table 2, even those which are likely to be projection pairs and contain galaxies without morphological distortions.

similar values for the merger fraction $f_{\text {merge }}$, as illustrated in Table 3: the merger fraction is $0.023 \pm 0.007$ and $0.021 \pm 0.007$, respectively, for $M_{\text {cut }} \geqslant 10^{9} M_{\odot}$ and $M_{\text {cut }} \geqslant 3.0 \times 10^{9} M_{\odot}$. Note that in computing the merger fraction, we only include the 20 distorted mergers listed in Table 2, and avoid the potential projection pairs of Types $2 \mathrm{a}$ and $2 \mathrm{~b}$ without signs of morphological distortions. ${ }^{19}$

While mergers may have played an important role in the evolution of cluster galaxies at earlier times (e.g., $z>2$ ), hierarchical models (e.g., Gottloeber et al. 2001; Khochfar \& Burkert 2001) predict that the merger fraction in dense clusters falls more steeply at $z<1$ than the field merger fraction. As a result, at $z<0.3$, the merger fraction for intermediate-mass cluster galaxies is predicted to be quite low (typically below $5 \%$ ). The low merger fraction among intermediate-mass $\left(M=10^{9}\right.$ to a few $\times 10^{10} M_{\odot}$ ) galaxies in the A901/902 clusters is consistent with the latter prediction.

How are the mergers distributed among major mergers, minor mergers, and ambiguous cases that could be either major or minor mergers? The results are shown in Columns 8-10 of Table 3, based on the classification listed in Column 8 of Table 2. The estimated fractions of likely major mergers, likely minor mergers, and ambiguous cases are $0.01 \pm 0.004 \%$ (9/886), $0.006 \pm 0.003 \%(5 / 886)$, and $0.007 \pm 0.003 \%(6 / 886)$, respectively for $M_{\text {cut }} \geqslant 10^{9} M_{\odot}$. For $M_{\text {cut }} \geqslant 3.0 \times 10^{9} M_{\odot}$, the corresponding fractions are $0.013 \pm 0.005 \%$ (8/609), $0.008 \pm$ $0.004 \%(5 / 609)$, and $0.0 \%(0 / 609)$, respectively.

In the rest of this paper, we continue to work with a mass cut of $M_{\text {cut }} \geqslant 10^{9} M_{\odot}$, and the 20 distorted mergers (Table 2) applicable for this mass cut. However, where relevant, we cite many of our results for both of the mass cuts $\left(M_{\text {cut }} \geqslant 10^{9} M_{\odot}\right.$ and $M_{\text {cut }} \geqslant 3.0 \times 10^{9} M_{\odot}$ ) so that we can gauge the potential effect of incompleteness in tracing major and minor mergers.

\subsection{Frequency of Mergers in A901/902 from CAS}

The results of running CAS on the final classified sample of 886 intermediate-mass $\left(M_{*} \geqslant 10^{9} M_{\odot}\right)$ systems are shown in Table 4 and Figures 6 and 7. In Figure 6, the 20 mergers of Type $1,2 \mathrm{a}$, and $2 \mathrm{~b}$ are plotted in different symbols. For Type $2 \mathrm{~b}$ merger pairs, we plot the highest value of CAS $A$ found between the two galaxies in each system. Using the CAS merger criterion $(A>0.35$ and $A>S$ ) to identify mergers yields a merger

\footnotetext{
19 Even if we included all pairs of Types $2 \mathrm{a}$ and $2 \mathrm{~b}$, the merger fraction would still have similar values $(0.041 \pm 0.01$ and $0.033 \pm 0.01)$ for both mass cuts (Table 3).
}

Table 4

Visual Classes of Galaxies Satisfying the CAS Criterion $(A>0.35$ and $A>S)$

\begin{tabular}{lcc}
\hline \hline \multicolumn{1}{c}{\begin{tabular}{c} 
Visual Class \\
\multicolumn{1}{c}{$(1)$}
\end{tabular}} & $N_{\text {CAS-VC }}$ & \multicolumn{1}{c}{$f_{\text {CAS-VC }}$} \\
& $(2)$ & $(3)$ \\
\hline All Visual Types & 18 & 1.00 \\
\hline Merger & 11 & $11 / 18=0.61 \pm 0.2$ \\
Non-interacting Irr-1 & 5 & $5 / 18=0.28 \pm 0.1$ \\
Non-interacting Symmetric & 2 & $2 / 18=0.11 \pm 0.08$ \\
\hline
\end{tabular}

Notes. The table shows the visual classes (VCs) of the galaxies, which satisfy the CAS criterion $(A>0.35$ and $A>S)$, among the sample of intermediate-mass $\left(M_{*} \geqslant 10^{9} M_{\odot}\right)$ systems. Columns: (1) VC of galaxies satisfying the CAS criterion; (2) $N_{\mathrm{CAS}-\mathrm{VC}}$ : the number of galaxies with this VC satisfying the CAS criterion; (3) $f_{\mathrm{CAS}-\mathrm{VC}}$ : the fraction of all the galaxies satisfying the CAS criterion, which have this VC.

fraction $\left(f_{\text {CAS }}\right)$ in this sample of $18 / 886$ or $0.02 \pm 0.006$. For the more conservative sample with a mass cut $M_{\text {cut }} \geqslant 3 \times 10^{9} M_{\odot}$, $f_{\text {CAS }}$ is $7 / 609$ or $0.011 \pm 0.005$.

When citing the error on $f_{\text {CAS }}$, we take the largest of either the Poisson error or the systematic error $\left(\sigma_{\mathrm{CAS}}\right)$ in $f_{\mathrm{CAS}}$ due to the systematic errors in CAS $A$ and $S$. The systematic error, $\sigma_{\mathrm{CAS}}$, is calculated by taking the upper and lower bounds of $f_{\text {CAS }}$ based on $(A \pm$ error in $A$ ) and $(S \pm$ error in $S$ ). Specifically, these limits on $f_{\mathrm{CAS}}$ are found by using the criteria of $((A \pm$ error in $A)<(S \pm$ error in $S))$ and $((A \pm$ error in $A)>0.35)$.

At first sight, the CAS-based merger fractions $f_{\text {CAS }}$ for the two mass cuts $(0.020 \pm 0.006$ and $0.011 \pm 0.005$ for $M_{\text {cut }} \geqslant 10^{9} M_{\odot}$ and $M_{\text {cut }} \geqslant 3 \times 10^{9} M_{\odot}$, respectively) are not widely different from the merger fraction $f_{\text {merge }}$ based on visual classification $(0.023 \pm 0.007$ and $0.021 \pm 0.007$ for $M_{\text {cut }} \geqslant 10^{9} M_{\odot}$ and $M_{\text {cut }} \geqslant 3 \times 10^{9} M_{\odot}$, respectively; Section 4.1). However, the comparison of $f_{\text {CAS }}$ and $f_{\text {merge }}$ does not tell the whole story because the nature of the systems picked by the two methods can be quite different. The visual classes of the 18 systems, which satisfy the CAS criterion and are considered as mergers in the CAS system, are shown in Table 4. It turns out $7 / 18(39 \pm 14 \%)$ of these "CAS mergers" are visually classified as non-interacting systems. The results are illustrated in Table 4 and Figure 6. Figure 7 shows examples of these "contaminants": they tend to be dusty highly inclined systems and systems with low level asymmetries that seem to be caused by SF.

It is also useful to ask what fraction of the 20 systems visually identified as mergers satisfy the CAS criterion. We find that for $M_{\text {cut }} \geqslant 10^{9} M_{\odot}$, the CAS criterion only captures 11 of the 20 $(55 \pm 16 \%)$ of the visually classified mergers. These results are 


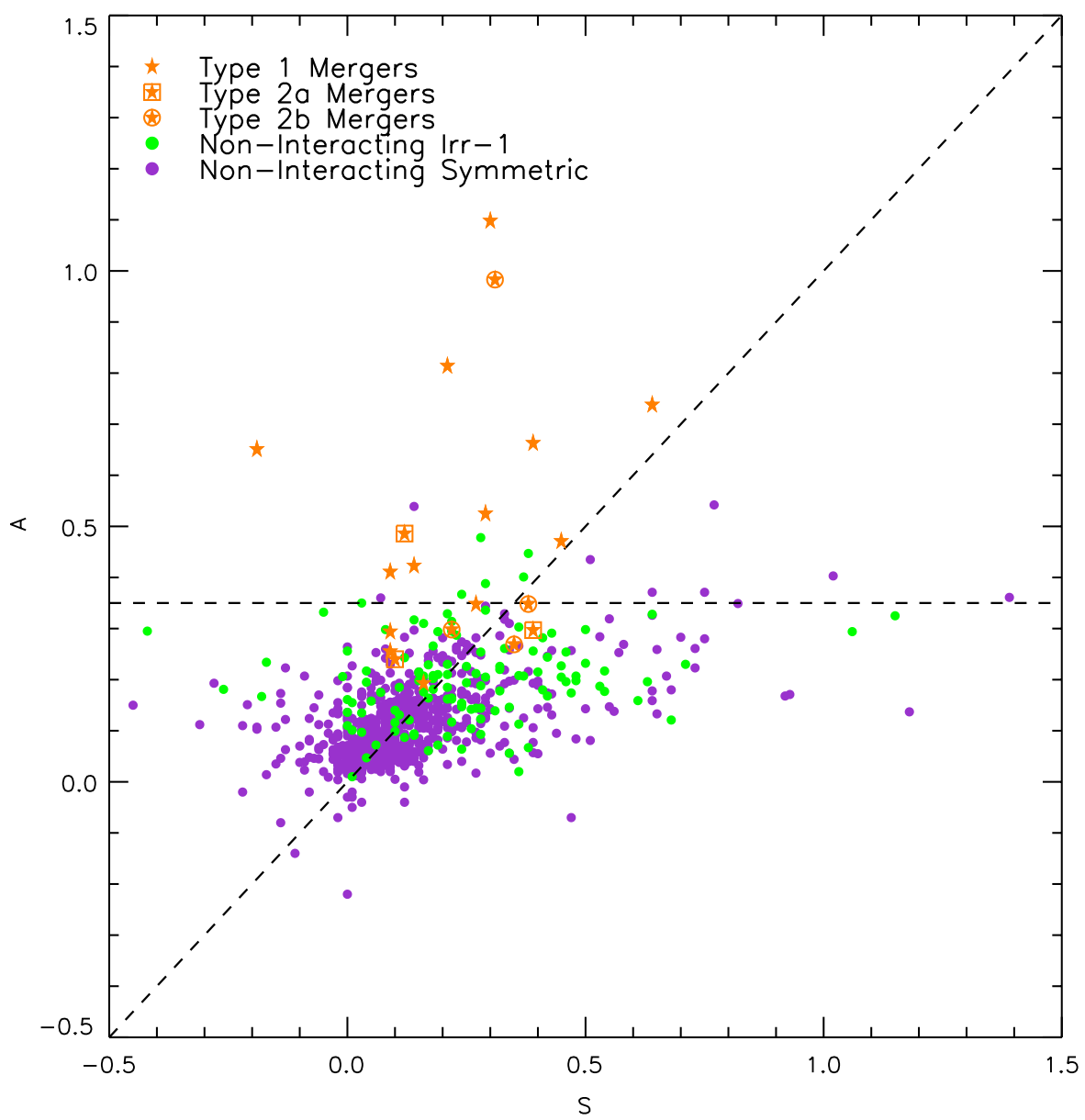

Figure 6. Results of running CAS on the sample of bright $\left(R_{\text {Vega }} \leqslant 24\right)$ intermediate-mass $\left(M_{*} \geqslant 10^{9} M_{\odot}\right)$ systems are shown. Galaxies with different visual classes (Merger, Non-interacting Irr-1, and Non-interacting Symmetric) are shown in the CAS $A$ vs. $S$ plane. We only plot here the 20 distorted mergers of Types $1,2 \mathrm{a}$, and $2 \mathrm{~b}$ in Table 2. The 18 galaxies satisfying the CAS merger criterion $(A>S$ and $A>0.35)$ lie on the upper left corner of the diagram. The CAS merger criterion recovers 11 of the $20(61 \% \pm 20 \%)$ of the galaxies visually classified as a merger. Furthermore, there is a significant level of contamination: 7/18 (39\% $\pm 14 \%)$ of the systems picked up by the CAS criterion are visually classified as Non-interacting Irr-1 and Non-interacting Symmetric.

(A color version of this figure is available in the online journal.)

illustrated in Figure 6. Figure 7 shows examples of merging galaxies missed by CAS. The missed mergers include galaxies with fainter outer tidal features, double nuclei where CAS puts the center between the nuclei, and pairs of very close connected galaxies.

It is also interesting to ask what percentage of the different types of mergers (Types $1,2 \mathrm{a}$, and $2 \mathrm{~b}$ ) does the CAS merger criterion recover. Figure 6 also shows the mergers divided up by merger Types $1,2 \mathrm{a}$ and $2 \mathrm{~b}$. Of the 13 mergers of Type 1, CAS recovers $9 / 13$ or $69 \% \pm 18 \%$. Of the three mergers of Type $2 \mathrm{a}$, CAS recovers $1 / 3$ or $33 \pm 28 \%$. Of the four mergers of Type $2 b$, CAS recovers $1 / 4$ or $25 \% \pm 22 \%$.

Since the CAS criterion is widely used to pick major mergers, it is also interesting to explore how well this criterion picks up the systems that we visually classified as major mergers (Table 2). We find that the CAS merger criterion picks up $6 / 9(67 \% \pm 20 \%)$ of the systems classified as major mergers. It is also interesting to note that the CAS criterion recovers $2 / 5$ $(40 \% \pm 23 \%)$ and $4 / 6(67 \% \pm 23 \%)$ of the systems classified respectively as minor mergers and ambiguous mergers.

\subsection{Distribution of Mergers}

In order to define different regions of the A901a, A901b, and A902 clusters, we computed the projected number density $n$ (Figure 8 ) for intermediate-mass $\left(M_{*} \geqslant 10^{9} M_{\odot}\right)$ galaxies as a function of clustocentric radius $R$ by assuming a spherical distribution. Note here that each galaxy is assigned to the cluster closest to it, and $R$ is measured from the center of that cluster.

We consider the cluster core to be at $R \leqslant 0.25 \mathrm{Mpc}$, as this is the region where the projected number density $n$ rises very steeply (Figure 8). The cluster virial radii are taken to be $\sim 1.2 \mathrm{Mpc}$, based on estimates from the DM maps derived from gravitational lensing by Heymans et al. (2008). Throughout this paper, we refer to the region at $0.25 \mathrm{Mpc}<R \leqslant 1.2 \mathrm{Mpc}$, between the cluster core and the cluster virial radius, as the "outer region of the cluster." The region outside the virial radius $(1.2 \mathrm{Mpc}<R \leqslant 2.0 \mathrm{Mpc})$ is referred to as the "outskirt region of the cluster." The core, outer region, and outskirt region are labeled in Figure 8.

As shown in Table 5, the A901 clusters have central galaxy number densities $(n)$ in the core region of 1000-1600 galaxies $\mathrm{Mpc}^{-3}$. These are lower than that of the rich Coma cluster and higher than that of the Virgo cluster.

Figure 9 shows the spatial distribution of the mergers visually identified among the sample of intermediate-mass $\left(M_{*} \geqslant\right.$ $10^{9} M_{\odot}$ ) galaxies in the A901a, A901b, and A902 clusters. We again consider here only the 20 distorted mergers listed in Table 2, for the reasons discussed in Section 4.1. We find that all the 20 mergers lie outside the cluster cores. We estimate that the number density ( $\left.n_{\text {merge }}\right)$ of mergers is $0,2.09$, and 

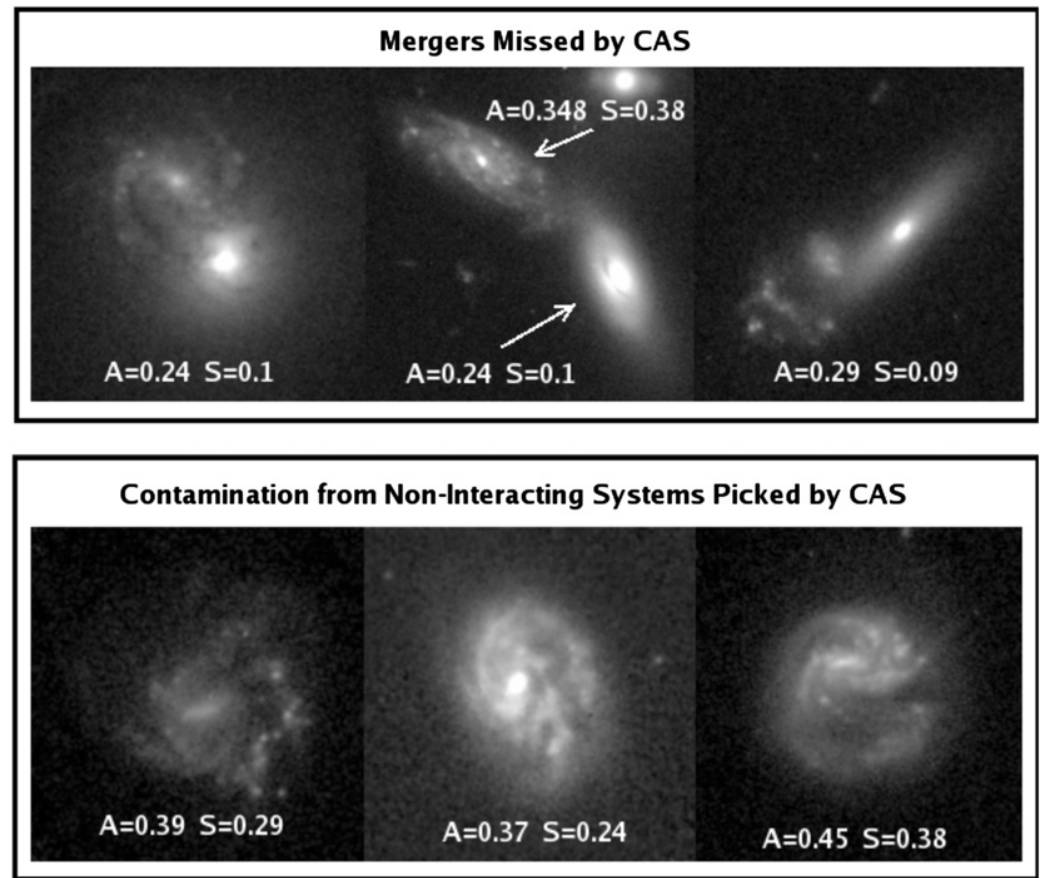

Figure 7. Top panel shows examples of mergers missed by the CAS merger criterion $(A>S$ and $A>0.35)$. Features of these systems include double nuclei, tidal bridges, and tidal debris. The lower panel shows some non-interacting contaminants picked up by the CAS merger criterion, due to small-scale asymmetries from SF, strong patchy dust lanes, and the absence of a clear center, all leading to a larger $A$ value.

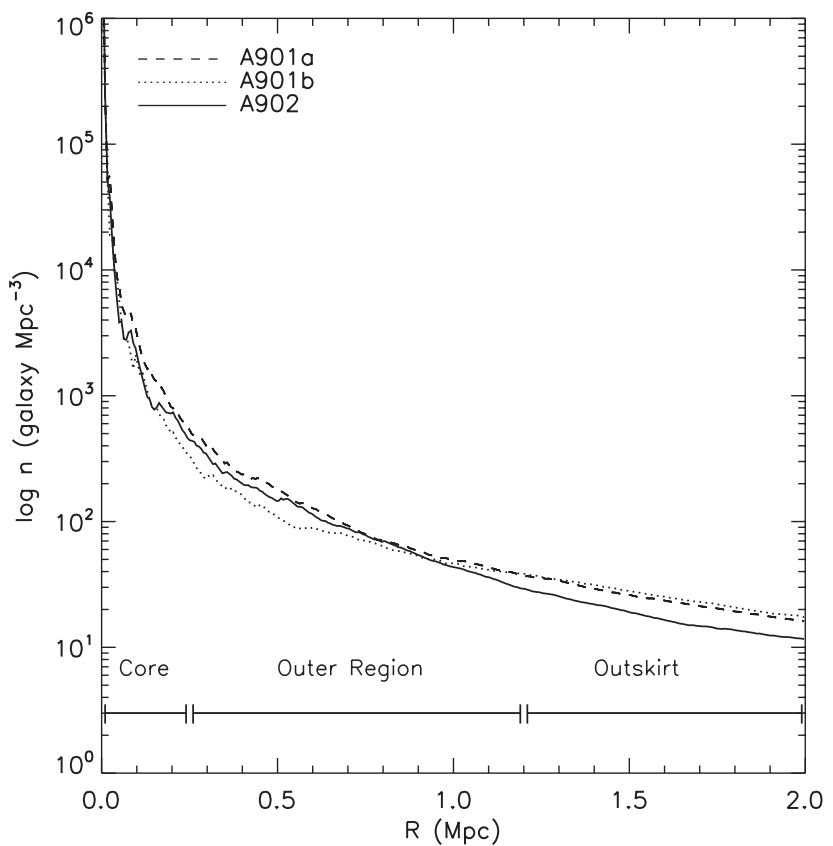

Figure 8. Azimuthally averaged projected number density $n$ of bright $\left(R_{\text {Vega }} \leqslant\right.$ $24)$ intermediate-mass $\left(M_{*} \geqslant 10^{9} M_{\odot}\right)$ galaxies in the sample is shown as a function of clustocentric radius which is shown for each cluster A901a/b and A902. We consider the cluster core to be at $R \leqslant 0.25 \mathrm{Mpc}$, as this is the region where the projected number density $n$ rises very steeply. We refer to the region which is located at $0.25 \mathrm{Mpc}<R \leqslant 1.2 \mathrm{Mpc}$ between the cluster core and the cluster virial radius as the outer region of the cluster. The region outside the virial radius (1.2 Mpc $<R \leqslant 2.0 \mathrm{Mpc})$ is referred to as the outskirt region of the cluster. The core, outer region, and outskirt region are labeled.

0.11 galaxies $\mathrm{Mpc}^{-3}$, respectively, in the core, outer region, and outskirt of the cluster (Table 6). When estimating $n_{\text {merge }}$ in the three regions, we divided the number of mergers by the volume of the core region, and the volume of a spherical shell in the outer region and outskirt of the clusters.
Table 5

Comparison of Projected Galaxy Number Densities $n$ and $\sigma_{\text {gal }}$

\begin{tabular}{lccc}
\hline \hline System & $\begin{array}{c}\text { Core } \\
R \leqslant 0.25 \mathrm{Mpc} \\
\left(\mathrm{galaxies} \mathrm{Mpc}^{-3}\right)\end{array}$ & $\begin{array}{c}\text { Outskirt } \\
R>1.2 \mathrm{Mpc} \\
\left(\mathrm{galaxies} \mathrm{Mpc}^{-3}\right)\end{array}$ & $\begin{array}{c}\sigma_{\text {gal }} \\
\left(\mathrm{km} \mathrm{s}^{-1}\right)\end{array}$ \\
\hline Virgo $^{\mathrm{a}}$ & 360 & 75 & $400-750$ \\
Coma $^{\mathrm{b}}$ & 10000 & 400 & $\sim 900$ \\
${\mathrm{~A} 901 \mathrm{a}^{\mathrm{c}}}^{\mathrm{c}}$ & 1600 & 60 & $890 \pm 182$ \\
$\mathrm{~A} 901 \mathrm{~b}^{\mathrm{c}}$ & 1100 & 55 & $1189 \pm 266$ \\
A902 $^{\mathrm{c}}$ & 1000 & 40 & $792 \pm 176$ \\
Groups $^{\mathrm{d}}$ & $\sim 0.01$ & & $\sim 100$ \\
\hline
\end{tabular}

Notes. This table compares the projected galaxy number densities $n$ and galaxy velocity dispersions $\sigma_{\mathrm{gal}}$ in the A901a, A901b, and A902 clusters to those of groups and other clusters, such as Coma and Virgo. For the A901 clusters, the value of $n$ is computed using the sample of bright $\left(R_{\text {Vega }} \leqslant 24\right)$ intermediatemass $\left(M_{*} \geqslant 10^{9} M_{\odot}\right)$ galaxies. References for the values cited are: ${ }^{\text {a }}$ Binggeli et al. (1987); ${ }^{\mathrm{b}}$ The \& White (1986); ${ }^{\mathrm{c}}$ number densities are from this work, and the average local velocity dispersions are from M. E. Gray et al. (2010, in preparation); ${ }^{\mathrm{d}}$ Tago et al. (2008).

For mergers and non-interacting galaxies, we compute the minimum distance to the nearest cluster center (A901a, A901b, and A902), and the local values of various environmental parameters, such as the local galaxy surface density $\Sigma_{10}$, the local DM mass surface density $\kappa$, and the relative local ICM density. Following Wolf et al. (2005) and Gilmour et al. (2007), we define and compute the local galaxy surface density $\left(\Sigma_{10}\right)$ as the number of galaxies per $(\mathrm{Mpc} / \mathrm{h})^{-2}$ that are in an annulus bracketed by two circles whose radii are equal to the average distances to the 9th and 10th nearest neighbor. The DM mass surface density $(\kappa)$ is computed locally using the coordinates of each galaxy on the weak lensing map provided by Heymans et al. (2008). The relative local ICM counts are measured at each galaxy position using the X-ray map from Grey et al. (2010, in preparation) and Gilmour et al. (2007). Figure 10 shows these 


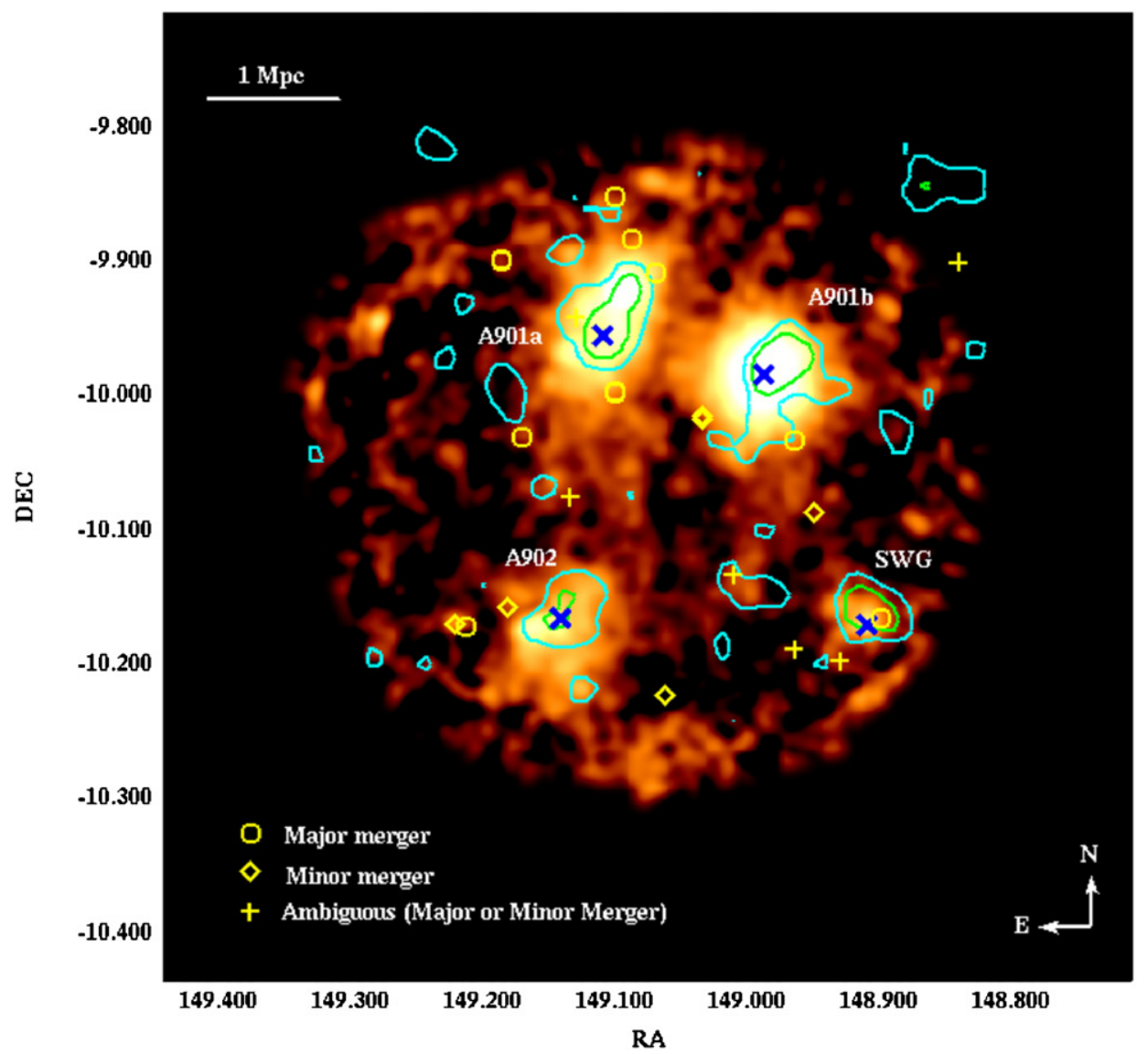

Figure 9. Distribution of visually classified mergers (coded as yellow diamonds, circles, and crosses) among the final sample of bright $\left(R_{\text {Vega }} \leqslant 24\right)$ intermediate-mass $\left(M_{*} \geqslant 10^{9} M_{\odot}\right.$ galaxies) is shown, overlaid on the ICM density map (yellow-orange scale). We only plot here the final sample of 20 distorted mergers, shown with starred identification numbers in Table 2. Following Heymans et al. (2008), we show DM masses in terms of the signal-to-noise of the weak lensing detection. The cyan and green contours enclose roughly $6 \mathrm{hr}^{-1} 10^{13} M_{\odot}$ and $3.5 \mathrm{hr}^{-1} 10^{13} M_{\odot}$ for A901a and A901b, and roughly $3 \mathrm{hr}^{-1} 10^{13} M_{\odot}$ and $1.5 \mathrm{hr}^{-1} 10^{13} M_{\odot}$ for the lower mass A902 and South West Group (SWG). The different symbols represent major mergers (circles), minor mergers (diamonds), and ambiguous major or minor merger cases (crosses), identified in Section 3.2. All mergers are located in the outer region and outskirt of each cluster $(0.25 \mathrm{Mpc}<R \leqslant 2 \mathrm{Mpc})$.

(A color version of this figure is available in the online journal.)

local environmental parameters as a function of the minimum distance to the cluster center for mergers and non-interacting galaxies. Since the mergers lie outside the cluster core $(0.25 \mathrm{Mpc}$ $<R \leqslant 2 \mathrm{Mpc}$ ), they are associated with low values of $\kappa$ and intermediate values of $\Sigma_{10}$, and ICM density (Figure 10).

\subsection{What Accounts for the Distribution of Mergers?}

The timescale for collisions and close encounters is given by

$$
t_{\mathrm{coll}}=\frac{1}{n \sigma_{\mathrm{gal}} A},
$$

where $n$ is the galaxy number density, $\sigma_{\text {gal }}$ is the galaxy velocity dispersion, $A$ is the collisional cross section $\left(\pi f\left(2 r_{\mathrm{gal}}\right)^{2}\right), r_{\mathrm{gal}}$ is a typical galaxy radius, and $f$ is the gravitational focusing factor (Binney \& Tremaine, 1987). Since the average velocity dispersion in clusters is high, the value for $f$ will be low and we will assume it to be on the order of unity. Thus,

$$
\begin{aligned}
t_{\text {coll }} \sim & 800 \times\left(\frac{n}{10^{3} \mathrm{Mpc}^{-3}}\right)^{-1} \times\left(\frac{\sigma}{10^{3} \mathrm{~km} \mathrm{~s}^{-1}}\right)^{-1} \\
& \times\left(\frac{r_{\text {gal }}}{10 \mathrm{kpc}}\right)^{-2} \mathrm{Myr} .
\end{aligned}
$$

We compute $n$ using the same method as in Section 4.3 for the core, and use spherical shells to compute $n$ in the outer region and cluster outskirt for our sample of $R_{\text {Vega }} \leqslant 24$, intermediate-mass $\left(M_{*} \geqslant 10^{9} M_{\odot}\right)$ galaxies. The local galaxy velocity dispersion profiles for A901a/b, A902, and South West Group (SWG) from kinematic modeling using $\sim 4202 \mathrm{dF}$ redshifts (M. E. Gray et al. 2010, in preparation) are shown in Figure 11, and the average velocity dispersions $\sigma_{\text {gal }}$ are shown in Table 5. The central galaxy velocity dispersion within the cores $(R<0.25 \mathrm{Mpc})$ of A901a,b and A902 typically range from 700 to $1000 \mathrm{~km} \mathrm{~s}^{-1}$. Beyond the cluster core, in the outer region $(0.25 \mathrm{Mpc}<R \leqslant 1.2 \mathrm{Mpc})$, the small number statistics leads to large error bars on the galaxy velocity dispersion, making it not viable to determine whether it remains high, drops, or rises. To estimate timescales, we take the average $\sigma_{\text {gal }}$ for A901/902 to be $\sim 800 \mathrm{~km} \mathrm{~s}^{-1}$ in three regions of the cluster. The timescales $\left(t_{\text {coll }}\right)$ for close encounters are shown in Table 6 and range from 0.765 to 13.5 to $135 \mathrm{Gyr}$ from the core $(R \leqslant 0.25 \mathrm{Mpc})$, to the outer region $(0.25 \mathrm{Mpc}<R \leqslant$ $1.2 \mathrm{Mpc})$, to the outskirt region $(1.2 \mathrm{Mpc}<R \leqslant 2.0 \mathrm{Mpc})$ of the cluster.

It may at first seem surprising that mergers do not preferentially lie in the cluster core, where the probability for close encounters is high due to the associated short value of the timescale $t_{\text {coll }}$ for collisions and close encounters. However, if $\sigma_{\mathrm{gal}}$ is large, a close encounter is unlikely to lead to a merger or a large amount of tidal damage (i.e., to strong morphological and kinematical distortions). This is because a large galaxy velocity dispersion will likely cause the energy $E$ of the reduced particle in a binary 

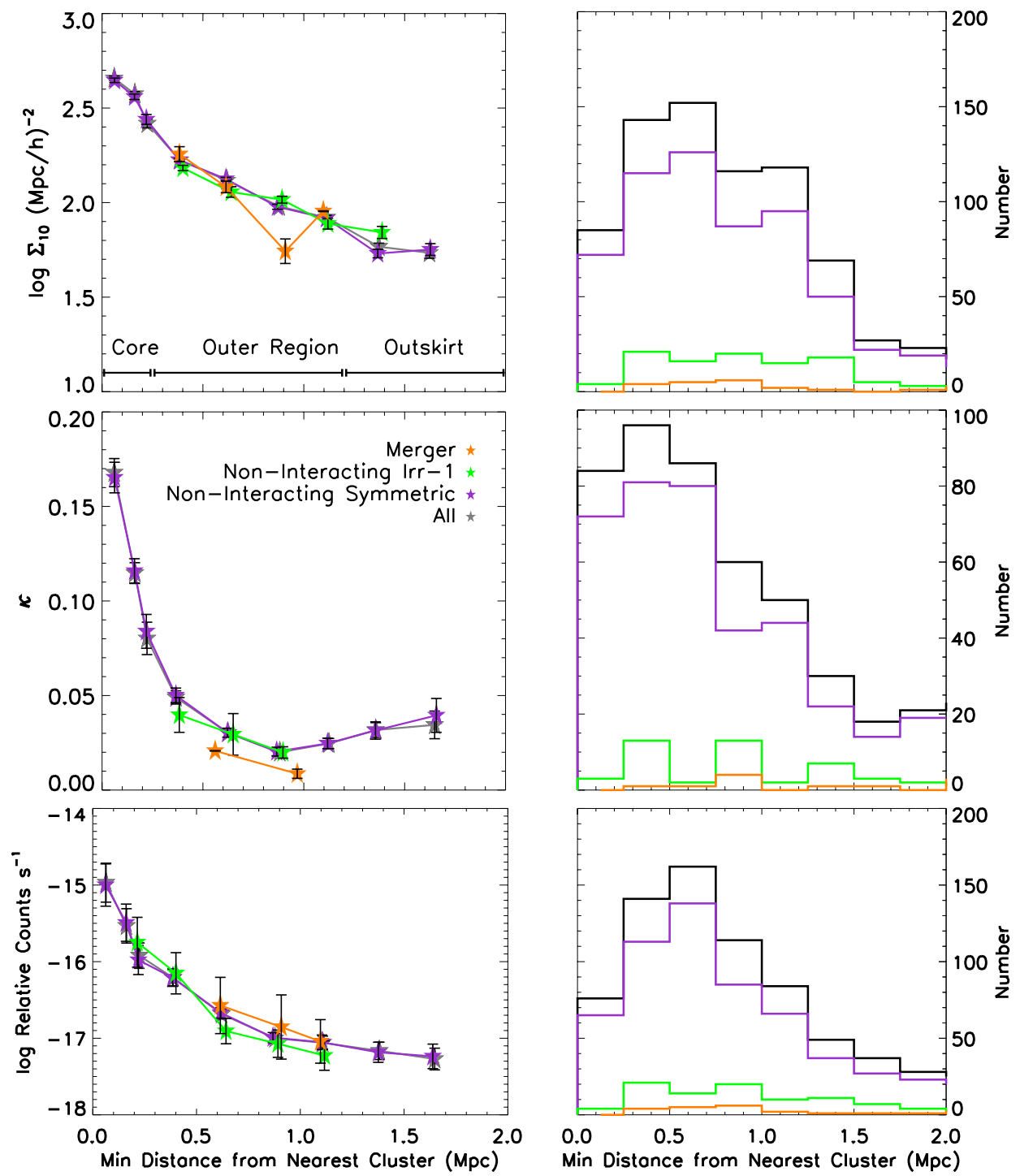

Figure 10. For mergers and non-interacting galaxies, the minimum distance to the nearest cluster center (A901a, A901b, A902) is plotted against the values of various local environmental parameters, such as the local galaxy surface density $\left(\Sigma_{10}\right)$ (top left), the local DM mass surface density $(\kappa)$ (middle left), and the relative local ICM density (bottom left). The panels on the right-hand side show the number of mergers and non-interacting galaxies which are found at different for each parameter. Mergers lie in the outer region and outskirt of the cluster $(0.25 \mathrm{Mpc}<R \leqslant 2 \mathrm{Mpc})$ and are associated with low values of $\kappa$ and intermediate values of $\Sigma_{10}$ and ICM density.

(A color version of this figure is available in the online journal.)

encounter to be positive, causing the orbit to become unbound (Binney \& Tremaine, 1987).

All merging systems lie at a projected clustocentric radius of $0.25 \mathrm{Mpc}<R \leqslant 2 \mathrm{Mpc}$, between the core and cluster outskirts, although in this region the timescale for collisions and close encounters is quite large. We suggest two possible reasons why strong interactions and mergers might populate the outer region and cluster outskirt. The first possibility is that the velocity dispersion of galaxies falls between the core and outskirt, due to the intrinsic velocity dispersion profile of the clusters. Many clusters show a flat velocity dispersion profile from $1 \mathrm{Mpc}$ outward, but several also show a declining profile (den Hartog \& Katgert 1996, Carlberg et al. 1997). In the latter cases, the velocity dispersions can fall from 1500 to $100 \mathrm{~km} \mathrm{~s}^{-1}$ from the cluster core to cluster outskirt. Since the local velocity dispersion profiles in the regions of interest in A901/902 (Figure 11) are based on a small number of galaxies and therefore large errors in $\sigma_{\text {gal }}$, we cannot determine if the velocity dispersion profile falls or remains flat. An additional difficulty is that the member galaxies of the neighboring clusters influence $\sigma_{\text {gal }}$ at $\sim 0.5 \mathrm{Mpc}$ for A901a/b and $\sim 1 \mathrm{Mpc}$ for A902.

The second possibility is that the mergers are part of groups or field galaxies that are being accreted along filaments by the A901/902 clusters. This would be in line with the idea that clusters continuously grow by mergers and accretion of groups (e.g., Zabludoff \& Franx 1993; Abraham et al. 1996; Balogh et al. 2000). Groups have lower galaxy velocity dispersion (typically below $300 \mathrm{~km} \mathrm{~s}^{-1}$ ) than clusters, as shown in Table 6 . Furthermore, simulations show that merger rates are highest as field and group galaxies accrete into cluster along cosmological filaments (see below; van Kampen \& Katgert 1997; Figure 12).

To further quantify the possibility of groups accreting into the A901/A902 clusters, we compare the data to model predictions from simulations of the STAGES A901/A902 supercluster (E. van Kampen et al. 2010, in preparation). The simulation aims to reproduce the environment as observed for A901/A902 by using constrained initial conditions that produce clusters with overall properties similar to those of A901, A902, and the 


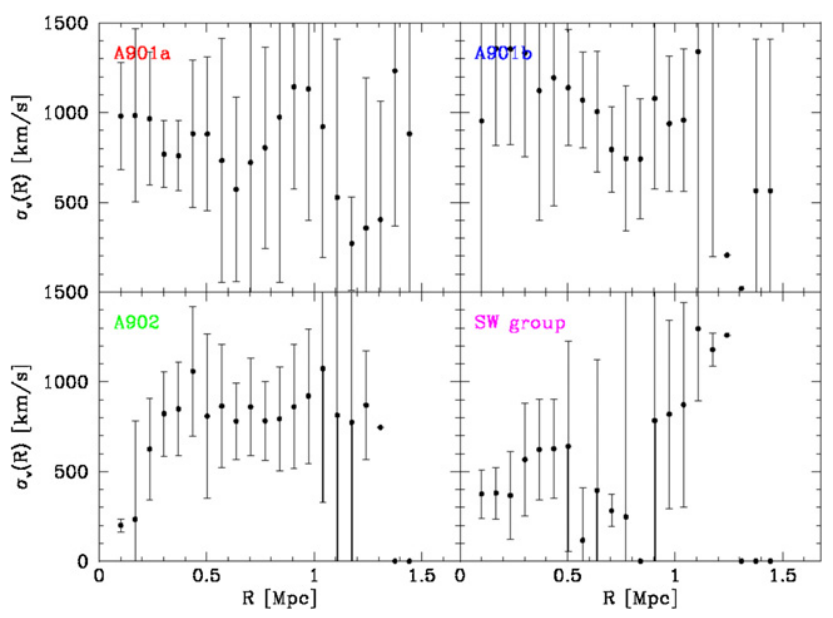

Figure 11. Local velocity dispersion profiles for A901a, b and A902 clusters, and associated SWG from kinematic modeling using the $\sim 4202 \mathrm{dF}$ redshifts (M. E. Gray et al. 2010, in preparation). The central galaxy velocity dispersion within the cores $(R<0.25 \mathrm{Mpc})$ of A901a,b and A902 typically range from 700 to $1000 \mathrm{~km} \mathrm{~s}^{-1}$. Outside the cluster core, in the outer region $(0.25 \mathrm{Mpc}$ $<R \leqslant 1.2 \mathrm{Mpc}$ ), the small number statistics leads to large error bars on the galaxy velocity dispersion, making it not viable to determine whether it remains high or drops.

neighboring A907 and A868. The simulation box is large enough to contain all these, and hence reproduce the overall largescale density and velocity fields similar to what is observed. A phenomenological galaxy formation model is then used to simulate the galaxy population in the same box, allowing us to select mock galaxies above a certain luminosity or stellar mass cut, as done in the observed data set. A stellar mass cut of $M_{*} \geqslant 10^{9} M_{\odot}$ is applied in the simulations, corresponding to the mass cut in the observational sample. The solid lines in Figure 12 show the predicted number density $(n)$ and fraction $(f)$ of galaxy mergers with mass ratio $M_{1} / M_{2}>1 / 10$, as a function of environment, as characterized by the local overdensity $\left(\delta^{\mathrm{G}}\right)$. The latter is calculated by smoothing the density of DM halos with a Gaussian of width $0.4 \mathrm{Mpc}$ to take out the effect of individual galaxies. Typical values of $\delta^{\mathrm{G}}$ are $\sim 10-100$ for group overdensities, $\sim 200$ at the cluster virial radius, and $\gtrsim 1000$ in the core of rich clusters.

Typically, in the simulations, as field and group galaxies fall into a cluster along filaments, the coherent bulk flow enhances the galaxy density and causes galaxies to have small relative velocities, thus leading to a high probability for mergers at typical group overdensities. Closer to the cluster core, galaxies show large random motions rather than bulk flow motion, leading to a large galaxy velocity dispersion, and a sharp drop in the probability of mergers.

In order to compare the data to simulation results, we use the number $\left(N_{\text {merge }}\right)$ and fraction $\left(f_{\text {merge }}\right)$ of mergers of mass ratio $\geqslant 1 / 10$, which we computed in Section 4.1. The solid curve in Figure 12 shows the merger fraction and number density predicted by the simulations as a function of overdensity. The three dashed lines in Figure 12 show the estimated range in the observational number density ( $\left.n_{\text {merge }}\right)$ and fraction $\left(f_{\text {merge }}\right)$ of mergers in the three different regions (the core, the outer region, and the outskirt) of the A901/902 clusters, as defined in Section 4.3. The points at which the dashed lines cross or approach the solid curve tell us the typical overdensities at which we expect to find such merger fraction or merger number densities in the simulations. It can be seen that the low merger density seen in the core region of the cluster corresponds to those expected at typical cluster core overdensities. On the other

Table 6

Properties in Core, Outer Region, and Outskirt of A901/902 Clusters

\begin{tabular}{|c|c|c|c|}
\hline & $\begin{array}{c}\text { Cluster Core } \\
(R \leqslant 0.25 \mathrm{Mpc})\end{array}$ & $\begin{array}{c}\text { Cluster Outer Region } \\
(0.25 \mathrm{Mpc}<R \leqslant 1.2 \mathrm{Mpc})\end{array}$ & $\begin{array}{c}\text { Outskirt } \\
(1.2 \mathrm{Mpc}<R \leqslant 2.0 \mathrm{Mpc})\end{array}$ \\
\hline (1) $N$ & 85 & 533 & 195 \\
\hline (2) Volume $V\left(\mathrm{Mpc}^{3}\right)$ & 0.065 & 7.17 & 26.3 \\
\hline (3) $n\left(\mathrm{gal} \mathrm{Mpc}^{-3}\right)$ & 1307 & 74.3 & 7.41 \\
\hline (4) $\langle B-V\rangle$ & 0.839 & 0.764 & 0.739 \\
\hline (5) $\langle U-V\rangle$ & 1.25 & 1.08 & 1.03 \\
\hline (6) $t_{\text {coll }}(\mathrm{Gyr})$ & 0.765 & 13.5 & 135 \\
\hline (7) $N_{\text {non-int }}$ & 84 & 519 & 192 \\
\hline (8) $N_{\text {merger }}$ & 0 & 15 & 3 \\
\hline (9) $n_{\text {merger }}\left(\right.$ merger $\mathrm{Mpc}^{-3}$ ) & 0 & 2.09 & 0.11 \\
\hline$(10) f_{\text {merger }}$ & 0 & 0.028 & 0.015 \\
\hline (11) $N_{\text {major-merger }}$ & 0 & 8 & 0 \\
\hline (12) $n_{\text {major-merger }}\left(\right.$ merger $\mathrm{Mpc}^{-3}$ ) & 0 & 1.12 & 0 \\
\hline (13) $f_{\text {major-merger }}$ & 0 & 0.015 & 0.005 \\
\hline (14) $N_{\text {minor-merger }}$ & 0 & 5 & 0 \\
\hline (15) $n_{\text {minor-merger }}\left(\right.$ merger $\left.\mathrm{Mpc}^{-3}\right)$ & 0 & 0.70 & 0 \\
\hline (16) $f_{\text {minor-merger }}$ & 0 & 0.009 & 0 \\
\hline (17) $N_{\text {non-int-BC }}$ & 7 & 178 & 81 \\
\hline (18) $f_{\text {non-int-BC }}$ & 0.08 & 0.34 & 0.42 \\
\hline (19) $N_{\text {merger-BC }}$ & 0 & 11 & 3 \\
\hline (20) $f_{\text {merger-BC }}$ & 0 & 0.73 & 1.0 \\
\hline
\end{tabular}

Notes. This table shows galaxy properties and timescales in three different regions of the cluster: the core $(R \leqslant 0.25 \mathrm{Mpc})$, the outer region $(0.25 \mathrm{Mpc}<R \leqslant$ $1.2 \mathrm{Mpc}$ ) between the core and the cluster, and the outskirt region $(1.2 \mathrm{Mpc}<R \leqslant 2.0 \mathrm{Mpc})$. The values are computed based on the sample of bright $(R$ Vega $\leqslant 24)$ intermediate-mass $\left(M_{*} \geqslant 10^{9} M_{\odot}\right)$ systems. Rows: (1) the total number of galaxies; (2) volume of the region; (3) projected number density of galaxies; (4) mean $B-V$ rest-frame color; (5) mean $U-V$ rest-frame color; (6) Timescale for collisions or close encounters; (7) The number of non-interacting galaxies; (8) The number of mergers from Table 2; (9) projected number density of mergers; (10) fraction of mergers; (11)-(13): same as (8)-(10), but for major mergers; (14)-(16): same as (8)-(10), but for minor mergers; (17) the number of non-interacting galaxies on blue cloud; (18) fraction of non-interacting galaxies on blue cloud; (19) The number of mergers on blue cloud; (20) fraction of mergers on blue cloud. 


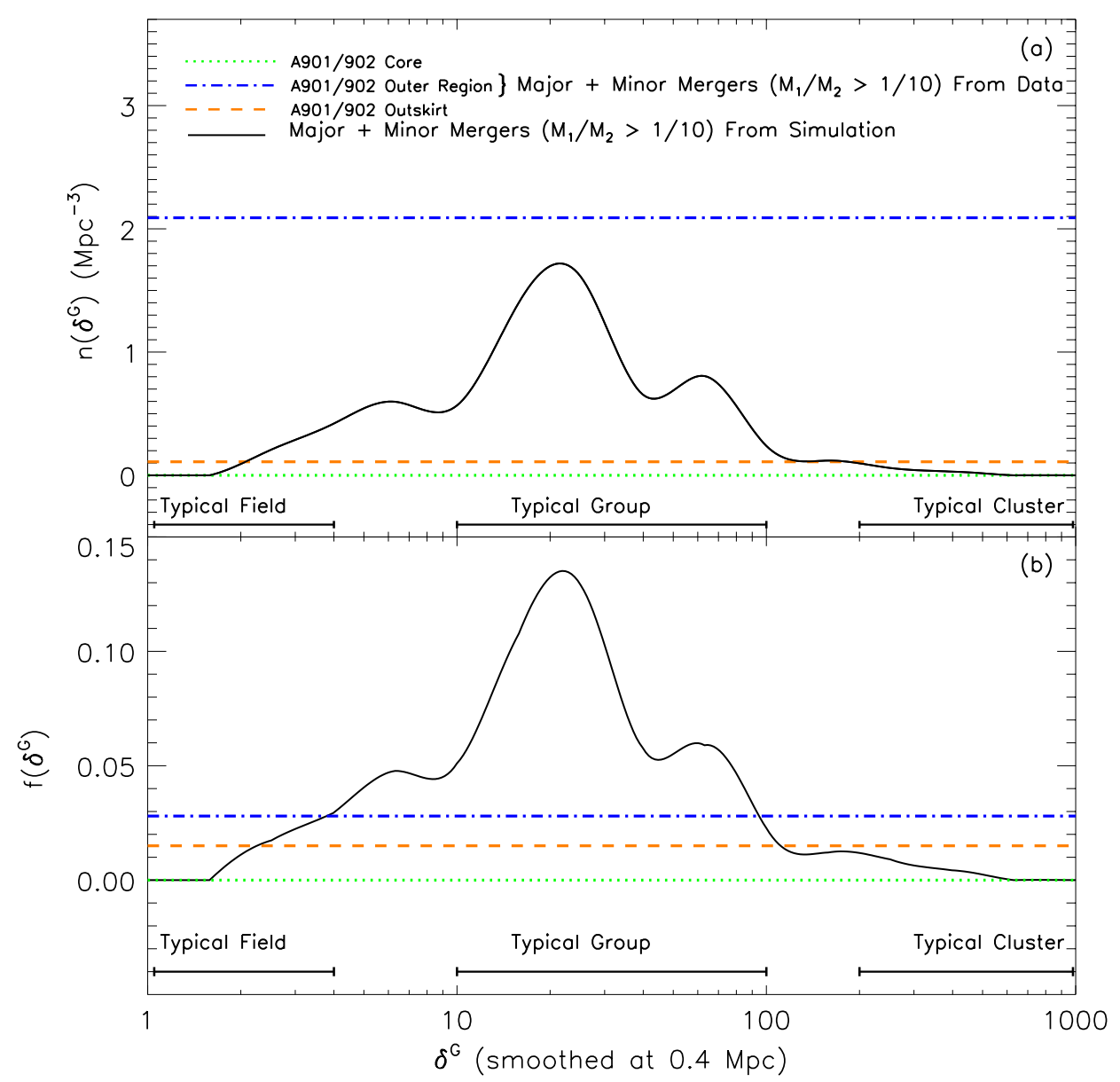

Figure 12. Comparison of the observed and theoretically predicted number density of mergers (top panel) and fraction of mergers (lower panel), as detailed in Section 4.4. The solid curve shows the predicted model number density of mergers $\left(n\left(\delta^{\mathrm{G}}\right)\right)$ and the fraction of mergers $\left(f\left(\delta^{\mathrm{G}}\right)\right)$ as a function of local overdensity $\left(\delta^{\mathrm{G}}\right)$, in the $N$-body simulations of the STAGES A901/902 supercluster (E. van Kampen et al. 2010, in preparation). The local overdensity is calculated by smoothing the density of DM halos with a Gaussian of width $0.4 \mathrm{Mpc}$ to takeout the effect of individual galaxies. Typical values of $\delta^{\mathrm{G}}$ are $\sim 10-100$ for group overdensities, $\sim 200$ at the cluster virial radius, and $>1000$ in core of rich clusters. In the models, as field and group galaxies fall into a cluster along filaments, the bulk flow enhances the galaxy density and causes galaxies to have small relative velocities, thus leading to a high probability for mergers at typical group overdensities. Closer to the cluster core, model galaxies show large random motions, producing a sharp drop in the probability of mergers. The three dashed lines show the observed number density $\left(n_{\text {merge }}\right)$ and fraction $\left(f_{\text {merge }}\right)$ of mergers in three different regions of the A901/902 clusters: the core, the outer region, and the outskirt. A stellar mass cut of $M>$ $10^{9} M_{\odot}$ is applied to both model and data. The points at which the three dashed lines cross or approach the solid curve tell us the typical overdensities at which we expect to find such merger fraction or merger number densities in the simulations. It can be seen that the low merger density seen in the core region (green dashed line) of the cluster correspond to those expected at typical cluster core overdensities. On the other hand, the larger merger fraction we observe between the cluster core and outer region $(0.25 \mathrm{Mpc}<R \leqslant 2 \mathrm{Mpc}$; blue dashed line) is close to those seen in typical group overdensities, in agreement with the scenario of cluster growth via accretion of group and/or field galaxies.

(A color version of this figure is available in the online journal.)

hand, the larger merger fraction we observe between the cluster core and outer region $(0.25 \mathrm{Mpc}<R \leqslant 2 \mathrm{Mpc})$ is close to those seen in typical group overdensities. Our results are in agreement with the above scenario, whereby the accretion of group and/or field galaxies along filaments, where they have low relative velocities and enhanced overdensities, leads to enhanced merger rates. We note that similar conclusions are reached if we perform the comparisons between data and simulations using the more conservative mass cut of $M_{*} \geqslant 3 \times 10^{9} M_{\odot}$, discussed in Section 4.1.

However, there are a couple of caveats when comparing our data with simulations. One caveat is that we directly compare the projected values of the number density or fraction of mergers from two-dimensional observations, to the predicted number density of mergers from three-dimensional simulations. Projection effects can introduce uncertainties in our observational estimate. The magnitude of the uncertainties due to projection effects depends on the detailed environment and will be in- vestigated in the full STAGES supercluster simulation data set (E. van Kampen et al. 2010, in preparation).

A further indirect evidence for group accretion stems from comparing semi-analytic galaxy catalogs to STAGES observations. R. Rhodes et al. (2010, in preparation) find an overabundance in galaxies in A902 compared to its mass. This could be explained by two or more galaxy groups in projection, consistent with the idea of group accretion.

\subsection{Comparison with Groups and Clusters at Different Epochs}

We first recapitulate our results on the visually based merger fraction $\left(f_{\text {merge }}\right)$ in the A901/902 clusters (Section 4.1). Among intermediate-mass $\left(M_{*} \geqslant 10^{9} M_{\odot}\right)$ systems, we find that the fraction $f_{\text {merge }}$ of systems which show evidence of a recent or ongoing merger of mass ratio $>1 / 10$ is $0.023 \pm 0.007$ (Table 3 ). Most of these mergers have $M_{\mathrm{V}} \sim-19$ to -22 and $L \leqslant L^{*}$ (see Figure 1). We also estimated that the fraction of likely major mergers, likely minor mergers, and ambiguous cases to 


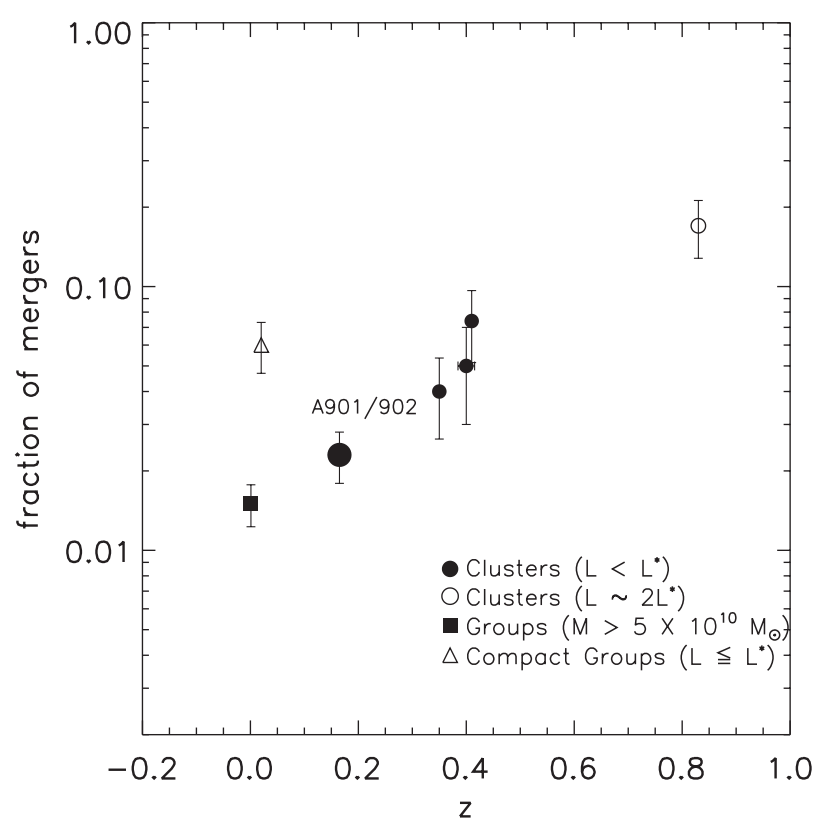

Figure 13. Comparisons of our observed fraction of mergers among intermediate-mass systems in the A901/902 clusters (large filled solid circle) with the results from other studies of clusters and groups. Filled circles are for cluster data points of intermediate luminosity ( $L<L^{*}$ and/or $M=10^{9}$ to a few $\left.\times 10^{10} M_{\odot}\right)$, and include the following studies in order of increasing redshift: this study of A901/902 (most systems have $M_{V} \sim-19$ to -22 and $M_{*}=10^{9}$ to a few $\left.\times 10^{10} M_{\odot}\right)$; Couch et al. $\left(1998 ; L<2 L^{*}\right)$, Oemler et al. (1997; lower limit of $f$ for $\left.M_{V}<-19\right)$, Dressler et al. (1994; $\left.M_{V}<-18.5\right)$. The van Dokkum et al. (1999) point at $z=0.83$ (open circle) is for a cluster sample of luminous $\left(M_{B} \sim-22\right.$ and $\left.L \sim 2 L^{*}\right)$ galaxies. The group point (filled square) is from McIntosh et al. (2008) for galaxy masses above $5 \times 10^{10} M_{\odot}$. The Hickson compact group point (open triangle), from Zepf et al. (1993), is for galaxies of luminosities $L \leqslant L^{*}$.

be $0.01 \% \pm 0.004 \%(9 / 886), 0.006 \% \pm 0.003 \%$ (5/886), and $0.007 \% \pm 0.003 \%$ (6/886), respectively.

Next, we compare our merger fraction in the A901/902 clusters with the published merger fraction in other clusters and group galaxies out to $z \sim 0.8$, over the last $7 \mathrm{Gyr}$ (Figure 13). These comparisons are difficult to make as different studies apply different luminosity or mass cuts. Furthermore, some studies consider only major mergers, while others consider all interacting galaxies, which are likely candidates for both major and minor interactions.

The variation in galaxy populations sampled at different epochs must also be kept in mind when comparing results at lower redshift with those out to $z \sim 0.8$. Low redshift samples typically sample a small volume and therefore tend to host only a small number of the brightest and most massive systems. Conversely, higher redshift magnitude-limited samples will suffer from Malmquist bias and tend to under-represent the faint $\left(L<L^{*}\right)$ galaxies.

There have been several studies of galaxy mergers and interactions in intermediate redshift clusters (Lavery \& Henry 1988; Lavery et al. 1992; Dressler et al. 1994; Oemler et al. 1997; Couch et al. 1998), but only few to date with high-resolution HST imaging. Couch et al. (1998) used WFPC/WFPC2 observations and spectroscopy of two clusters at $z \sim 0.3$ and $\sim 0.4$, focusing on galaxies with $R \sim 22.5$ or $L<L^{*}$. In their study, they classified $\sim 7 / 200$ or $\sim 3.5 \%$ galaxies to be merging based on separations and visible distortions. The merger fractions of these two intermediate redshift clusters are plotted in Figure 13.

It is also interesting to note that the merging systems in these two intermediate redshift clusters tend to be blue and preferentially located in the outskirt of the clusters. In fact, in the Couch et al. (1998) sample, $\sim 60 \%$ of the merging galaxies among $L<L^{*}$ systems are blue. The mergers in these two intermediate redshift clusters are similar in many ways to the mergers in the A901/902 clusters: the latter mergers lie in between the cluster core and outskirt $(0.25 \mathrm{Mpc}<R \leqslant 2 \mathrm{Mpc}$; Section 4.3) of the A901/902 clusters, and for the absolute magnitude range of $M_{\mathrm{V}} \sim-19$ to $-22,16 / 20$, or $80 \% \pm 18 \%$ of these mergers lie on the blue cloud (Section 4.6).

In a study by Dressler et al. (1994), based on WFPC images of a $z \sim 0.4$ cluster, the fraction of bright $\left(M_{V}<-18.5\right)$ galaxies identified as mergers was $10 / 135$. Another WFPC study of four clusters at $z=0.37-0.41$ by Oemler et al. (1997) placed a lower limit of $5 \%$ on the fraction of merging galaxies with $M_{V}<-19$ or $L \leqslant L^{*}$.

A high-redshift $(z=0.83)$ cluster was studied by van Dokkum et al. (1999) using HST WFPC2 images and spectroscopic redshifts for 80 confirmed members. They found a high fraction $(17 \%)$ of luminous $\left(M_{B} \sim-22 ; L \sim 2 L^{*}\right)$ systems, which exhibit signatures of ongoing mergers. These mergers were found to be red, bulge-dominated, and located in the outskirts of the cluster. A later study by Tran et al. (2005) used a spectroscopic follow-up to confirm that $15.7 \% \pm 3.6 \%$ of these galaxies were bound pairs with separations $\lesssim 30 \mathrm{hr}^{-1} \mathrm{kpc}$ and relative velocities $\lesssim 300 \mathrm{~km} \mathrm{~s}^{-1}$. These merging pairs are located outside the cluster centers, similar to the mergers in A901/ 902. However, the galaxy sample at high redshift is dominated by more luminous galaxies $\left(M_{B} \sim-22 ; L \sim 2 L^{*}\right)$ than the A901/902 sample where most galaxies have $M_{V} \sim-19.5$ to -22 (Figure 1). The number statistics for bright $\left(\sim 2 L^{*}\right)$ mergers in A901/902 are not robust enough to allow a direct comparison with the merger fraction cited in van Dokkum et al. (1999).

Finally, we look at the merger fraction in groups. A study by McIntosh et al. (2008) finds that $\sim 1.5 \%$ of massive $(M \geqslant 5 \times$ $10^{10} M_{\odot}$ ) galaxies in SDSS groups (with $M_{\text {halo }}>2.5 \times$ $\left.10^{13} M_{\odot}\right)$ are major mergers at $z$ of $0.01-0.12$. Most of the mergers involve two red sequence galaxies and they are located between 0.2 and $0.5 \mathrm{Mpc}$ from the group center. A study by McGee et al. (2008) also finds an enhancement in asymmetric bulge-dominated galaxies in groups, consistent with a higher probability for merging in the group environment. Additional evidence for mergers in groups is shown in a study of a supergroup at $z \sim 0.37$ by Tran et al. (2008), who report dry dissipationless mergers and signatures thereof in three of four brightest group galaxies.

A merger fraction of $6 \%$ was found by Zepf (1993) in Hickson compact groups at $z<0.05$, among systems with luminosities $L \leqslant L^{*}$. The merger fraction is significantly higher than that in SDSS groups. The increased merger fraction is expected since Hickson compact groups are different from loose groups: they have high number densities comparable to those in cluster cores, but low galaxy velocity dispersions. These two conditions provide an environment most favorable to strong tidal interactions and mergers, as argued in Section 4.4.

It is not straightforward to draw conclusions about the evolution of the merger fraction in cluster galaxies over $z \sim 0.05-1.0$ from the above studies because they sample different types of systems and different luminosity ranges. If we conservatively restrict ourselves to only studies of $L \leqslant L^{*}$ cluster galaxies, then we have four data points over $z \sim 0.17-0.4$, shown as solid filled circles in Figure 13. The mean value of the data points allows for evolution by a factor of $\sim 3.2$, in the merger fraction of $L \leqslant L^{*}$ cluster galaxies over $z \sim 0.17-0.4$. However, if 
one uses the full range of merger fractions allowed by the error bars on the data points, then we can admit a wider spectrum of scenarios, ranging from no evolution to evolution by a factor of $\sim 5$ over $z \sim 0.17-0.4$. Having additional deep, large-volume, high-resolution studies, which are based on larger samples with smaller error bars would help to separate between these scenarios, and thereby test hierarchical models of galaxy evolution.

As mentioned in Section 4.1, hierarchical models (e.g., Gottloeber et al. 2001; Khochfar \& Burkert 2001) predict that the merger fraction in dense clusters falls more steeply at $z<1$ than the field merger fraction, such that at $z<0.3$, the predicted merger fraction is quite low (typically below $5 \%$ ) among intermediate-mass cluster galaxies. The low merger fractions among intermediate-mass $\left(10^{9}\right.$ to a few $\left.\times 10^{10} M_{\odot}\right)$ or intermediate luminosities $\left(L<L^{*}\right)$ galaxies in the A901/902 clusters and other low-redshift clusters are consistent with these predictions, but we cannot yet test the predicted rate of evolution of the merger fraction in clusters with redshfit.

\subsection{Galaxies on the Blue Cloud and Red Sample}

We explore the properties of galaxies on the blue cloud and in the red sample among the final sample of 886 systems (20 mergers and 866 non-interacting galaxies; Section 3.2). The red sample was defined in Wolf et al. (2009) and contains both passively evolving spheroidal galaxies on the red sequence, as well as dusty red galaxies that lie between the red sequence and the blue cloud.

Figure 14 shows the rest-frame $U-V$ color plotted against stellar masses for galaxies of different visual classes: Mergers, Non-interacting Irr-1, and Non-interacting Symmetric (Section 3.2). The visual classes of galaxies on the blue cloud and in the red sample are shown in Table 7. As described in Section 4.1, we only consider the 20 distorted mergers listed in Table 2, and avoid the potential projection pairs without signs of morphological distortions. The 20 mergers are divided into 13 mergers of Type 1, 3 mergers of Type $2 \mathrm{a}$, and 4 mergers of Type $2 \mathrm{~b}$. For mergers of Type $2 \mathrm{~b}$, which are resolved into two galaxies with separate COMBO-17 colors, we plot the average $U-V$ color of the galaxies in the pair.

Out of the 886 visually classified systems in our sample, we find that $310 / 886$ or $35 \% \pm 7 \%$ lie on the blue cloud. Out of the 20 visually classified mergers with distortions, $16 / 20$ or $80 \% \pm 18 \%$ lie on the blue cloud (Table 7). Conversely, out of 866 non-interacting galaxies, $294 / 866$ or $34 \% \pm 7 \%$ are on the blue cloud. Thus, the fraction of mergers, which lie on the blue cloud $\left(f_{\text {merg-BC }}\right)$ is over two times larger than the fraction of non-interacting galaxies $\left(f_{\text {non-int-BC }}\right)$, which lie on the blue cloud. This implies that mergers and interacting galaxies are preferentially blue, compared to non-interacting galaxies.

The observed higher fraction of blue galaxies among mergers compared to non-interacting galaxies may be caused by several factors. It may be due to enhanced levels of unobscured SF in mergers (see Section 4.7), translating to bluer colors on average. It may also in part be the result of the mergers being part of accreted field and group galaxies (Section 4.4), which are bluer than the average cluster galaxy. It is also relevant to ask whether we are overestimating the fraction of blue galaxies among interacting systems due to the visibility timescale $t_{\text {vis }}$ of morphological distortions induced by interactions being longer in bluer galaxies than redder ones. While this is possible, it is non-trivial to correct for this effect because no direct unique relation exists between $t_{\mathrm{vis}}$ and color. As discussed in Section $4.1, t_{\mathrm{vis}}$ depends on the mass ratio of an interaction as well as the gas content. A higher gas content may result in a longer $t_{\mathrm{vis}}$ for certain gas distributions, but it can lead to either redder colors (e.g., enhanced level of dusty SF) or bluer colors (enhanced level of unobscured SF), compared to interacting systems.

\subsection{SF Properties of Interacting Galaxies}

This work uses SFRs based on UV data from COMBO-17 (Wolf et al. 2004) and Spitzer $24 \mu \mathrm{m}$ imaging (Bell et al. 2007). The unobscured $\mathrm{SFR}_{\mathrm{UV}}$ is derived using the $2800 \AA$ rest-frame luminosity $\left(L_{\mathrm{UV}}=1.5 v l_{v, 2800}\right)$ as described in Bell et al. (2005, 2007). The UV spectrum is dominated by continuum emission from massive stars and provides a good estimate of the integrated SFR from the younger stellar population in the wavelength range of $1216-3000 \AA$. The $\mathrm{SFR}_{\mathrm{IR}}$ is derived using the $24 \mu \mathrm{m}$ flux to construct the integrated IR luminosity $\left(L_{\mathrm{IR}}\right)$ over $8-1000 \mu \mathrm{m}$ following the methods of Papovich \& Bell (2002). The total SFR is derived using identical assumptions of Kennicutt (1998) from PEGASE assuming a 100 Myr old stellar population with constant SFR and a Chabrier (2003) IMF:

$$
\mathrm{SFR}_{\mathrm{UV}+\mathrm{IR}}=9.8 \times 10^{-11}\left(L_{\mathrm{IR}}+2.2 L_{\mathrm{UV}}\right) .
$$

The factor of 2.2 on the UV luminosity accounts for light being emitted longward of $3000 \AA$ and shortward of $1216 \AA$ by young stars. The total SFR accounts for both the dust-reprocessed (IR) and unobscured (UV) SFs.

We work with our final sample of 886 classifiable bright massive $\left(M_{*} \geqslant 10^{9} M_{\odot}\right)$ systems, and only consider here the 20 distorted mergers in Table 2 . All of these systems have UV-based SFR from COMBO-17 observations. Of this sample, $\sim 11 \%$ (94/886) were not observed with Spitzer, 23\% (206/886) were observed and detected at $24 \mu \mathrm{m}$, while the rest had no detection at the $\sim 4 \sigma$ depth of $83 \mu \mathrm{Jy}$.

The UV-based SFR $\left(\mathrm{SFR}_{\mathrm{UV}}\right)$ versus stellar mass is plotted in Figure 15 for all 886 systems. The $S F R_{U V}$ ranges from $\sim 0.01$ to $14 M_{\odot} \mathrm{yr}^{-1}$. The UV-based SFR represents a lower limit to the total SFR for galaxies on the blue cloud and most star-forming galaxies on the red sample. However, for some old red galaxies, the $\mathrm{SFR}_{\mathrm{UV}}$ may overestimate the true SFR as the UV light from such systems may not trace massive OB stars, but rather low- to intermediate-mass stars.

Figure 16 shows the UV+IR-based SFR (SFR $\left.{ }_{U V+I R}\right)$, which ranges from $\sim 0.2$ to $9 M_{\odot} \mathrm{yr}^{-1}$. For the 206 galaxies that were observed and detected at $24 \mu \mathrm{m}$, the implied UV+IR-based SFR is plotted as stars in the lower panel of Figure 16. For the 586 galaxies that are observed but undetected with Spitzer, we use the $24 \mu \mathrm{m}$ detection limit as an upper limit on the $24 \mu \mathrm{m}$ flux. The corresponding upper limit on the UV+IR-based SFR is plotted as inverted triangles in the lower panel of Figure 16, and included in the calculation of the average UV+IR-based SFR, plotted in the middle panel.

In a cluster environment, the competition between processes that enhance the SFR and those that depress the SFR, ultimately determine the average SFR of cluster galaxies. The first class of processes are strong close interactions: tidal and mergers (e.g., Toomre \& Toomre 1972), and harassment (e.g., Moore et al. 1996), which refers to the cumulative effect of weak interactions. The second class of processes include ram pressure stripping of cold gas out of the galaxy by the hot ICM (e.g., Gunn \& Gott 1972), and strangulation (e.g., Larson et al. 1980; Balogh et al. 2000).

For the few mergers (orange line) present, the average $\mathrm{SFR}_{\mathrm{UV}}$ is typically enhanced by an average modest factor of $\sim 2$ 

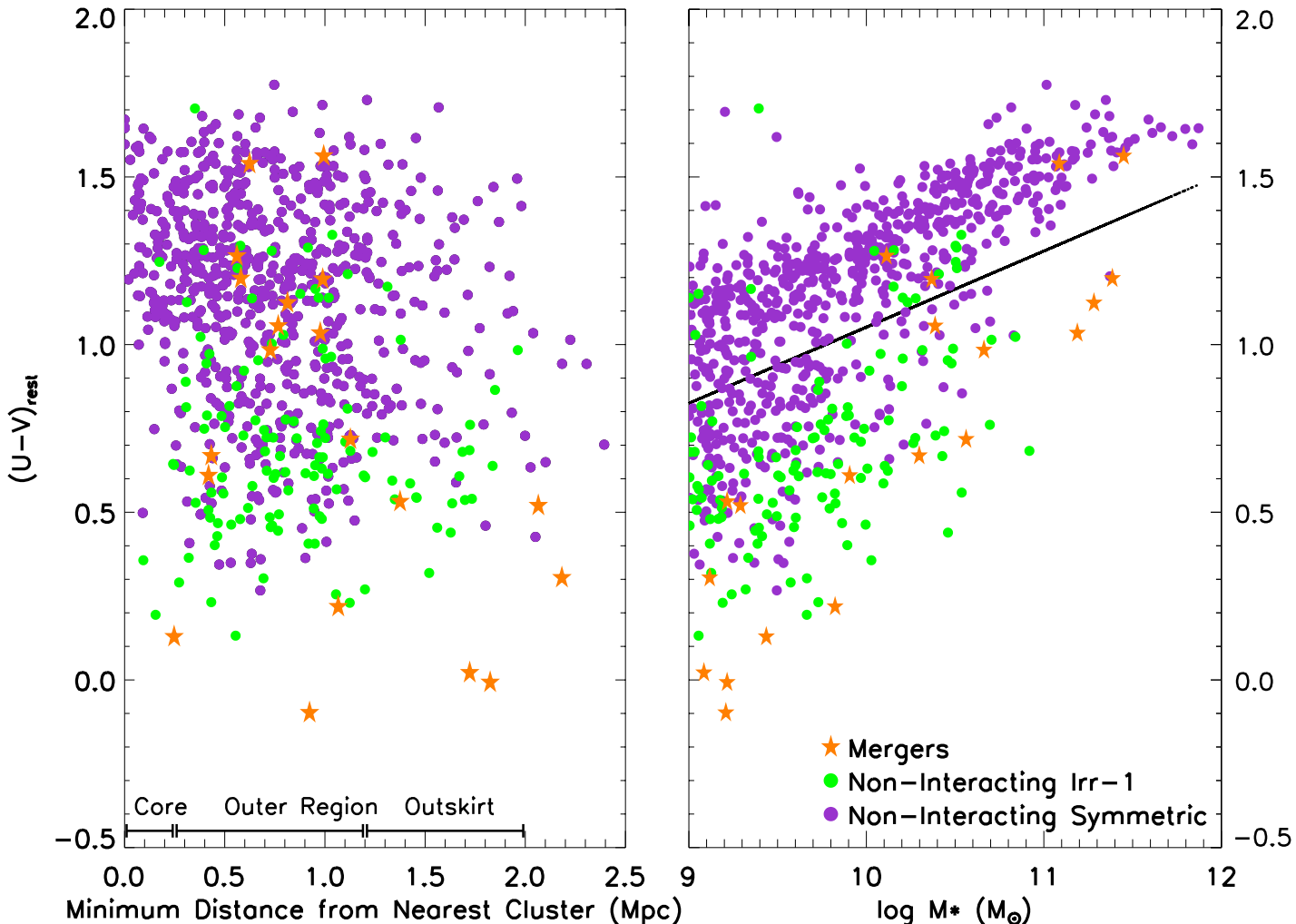

Figure 14. Left: the rest-frame $U-V$ color is plotted against the minimum distance from the nearest cluster center, for systems of different visual classes (Merger, Non-interacting Irr-1, and Non-interacting Symmetric). We only consider here the 20 distorted mergers in Table 2, split into 13 mergers of Type 1, 3 mergers of Type 2a, and 4 mergers of Type $2 \mathrm{~b}$. For mergers of Type $2 \mathrm{~b}$, which are resolved into two galaxies with separate COMBO-17 colors, we plot the average $U-V$ color of the galaxies in the pair. Right: the rest-frame $U-V$ color is plotted against stellar masses. The black solid lines separate the blue cloud and red sample. For $M \geqslant 10^{9} M_{\odot}$ systems, we find that $80 \% \pm 18 \%$ (16/20) of mergers lie on the blue cloud, compared to (294/866) or $34 \% \pm 7 \%$ of the non-interacting galaxies. Thus, mergers and interacting galaxies are preferentially blue compared to non-interacting galaxies.

(A color version of this figure is available in the online journal.)

Table 7

Visual Classes of Galaxies on the Blue Cloud and Red Sample

\begin{tabular}{lcccc}
\hline \hline \multicolumn{1}{c}{\begin{tabular}{c} 
Visual Class \\
\multicolumn{1}{c}{$(1)$}
\end{tabular}} & $\begin{array}{c}N_{\text {blue }} \\
(2)\end{array}$ & $\begin{array}{c}f_{\text {blue }} \\
(3)\end{array}$ & $\begin{array}{c}N_{\text {red }} \\
(4)\end{array}$ & $\begin{array}{c}f_{\text {red }} \\
(5)\end{array}$ \\
\hline (1) All & 310 & 1.00 & 576 & 1.00 \\
(2) Mergers & 16 & $16 / 310=0.052 \pm 0.02$ & 4 & $4 / 576=0.007 \pm 0.004$ \\
(2a) Likely Major Mergers & 6 & $6 / 310=0.02 \pm 0.009$ & 3 & $3 / 576=0.005 \pm 0.003$ \\
(2b) Likely Minor Mergers & 4 & $4 / 310=0.01 \pm 0.007$ & 1 & $1 / 576=0.002 \pm 0.002$ \\
(2c) Ambiguous Major/Minor & 6 & $6 / 310=0.02 \pm 0.009$ & 0 & 0 \\
(3) Non-interacting Irr-1 & 105 & $105 / 310=0.34 \pm 0.07$ & 18 & $18 / 576=0.033 \pm 0.01$ \\
(4) Non-interacting Symmetric & 189 & $189 / 310=61 \pm 13$ & 554 & $554 / 576=0.96 \pm 19$ \\
\hline
\end{tabular}

Notes. Results are shown for the sample of 886 bright $\left(R_{\mathrm{Vega}} \leqslant 24\right)$ intermediate-mass $\left(M_{*} \geqslant 10^{9} M_{\odot}\right)$ visually classifiable systems. The table shows how galaxies in the blue cloud and red sample are split among the different visual classes (Mergers, Non-interacting Irr-1, and Non-interacting Symmetric) discussed in Section 4.6. Columns: (1) Visual classes: these include Mergers, Non-interacting Irr-1, Non-interacting Symmetric, listed in rows 2, 3, and 4. We only consider the 20 morphologically distorted mergers listed in Table 2 . These include 13 mergers of Type 1, 3 mergers of Type 2a, and 4 mergers of Type $2 b$. Rows $2 a, 2 b$, and $2 c$ show how the mergers are split into likely major mergers, likely minor mergers, and ambiguous cases that could be either (Section 3.2). (2) $N_{\text {blue }}$ : the number of galaxies on the blue cloud; (3) $f_{\text {blue }}$ : fraction of blue cloud galaxies, which belong to a given visual class; (4) $N_{\text {red }}$ : the number of galaxies in the red sample; $(5) f_{\text {red }}$ : fraction of red sample galaxies, which belong to a given visual class.

compared to the both Non-interacting Symmetric and Irr-1 galaxies (purple, green, and black lines). Similarly, the UV+IRbased SFR ( $\mathrm{SFR}_{\mathrm{UV}+\mathrm{IR}}$; and Figure 16) of merging galaxies is typically enhanced by only an average factor of $\sim 1.5$ compared to the Non-interacting Symmetric galaxies (purple line) and to all Non-interacting galaxies (i.e., Symmetric + Irr-1; black line). We note that a similar modest enhancement in the average
SFR, by a factor of $1.5-2$ is also found in mergers in the field over $z \sim 0.24-0.80$ by Jogee et al. $(2008,2009)$. This modest enhancement is consistent with the theoretical predictions of di Matteo et al. (2007; see their Figure 10), based on a recent statistical study of several hundred simulated galaxy collisions. Modest SFR enhancements are also seen in galaxy pair studies in the field (Barton et al. 2000, 2003; Lin et al. 2004; Ellison 


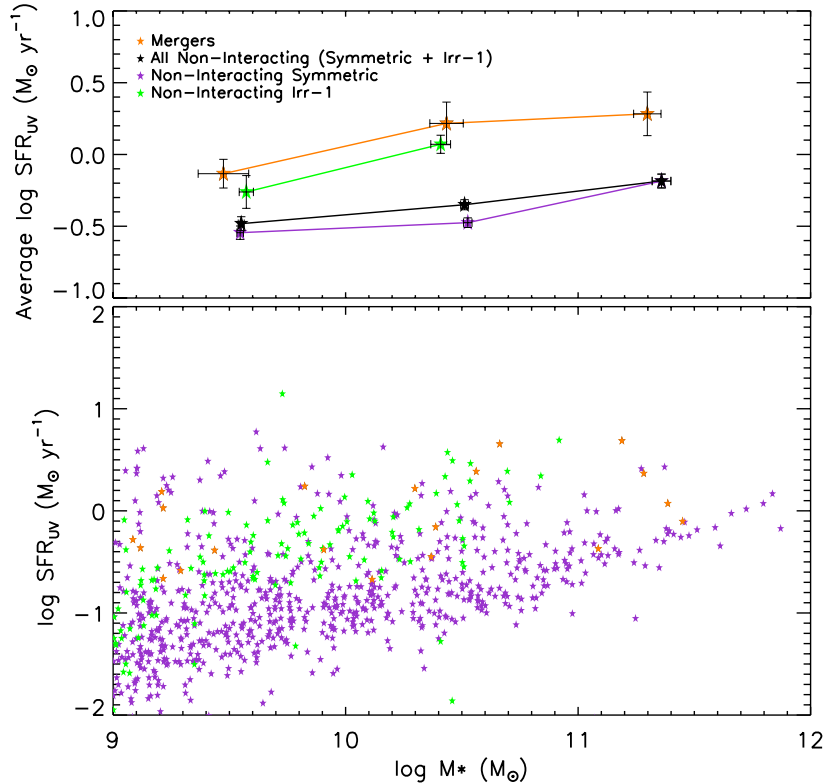

Figure 15. Bottom panel: the UV-based SFR is plotted against stellar mass for the sample of 886 bright intermediate-mass $\left(M_{*} \geqslant 10^{9} M_{\odot}\right)$ visually classifiable systems. Systems are coded according to their visual classes: Merger, Noninteracting Irr-1, and Non-interacting Symmetric. We only consider here the 20 distorted mergers in Table 2. Top panel: the average UV-based SFR is plotted against stellar mass for galaxies of different visual classes. For the few mergers galaxies present (orange line), the average $\mathrm{SFR}_{\mathrm{UV}}$ is enhanced by at most an average factor of $\sim 2$ compared to the Non-interacting Symmetric galaxies (purple line) and to all Non-interacting galaxies (i.e., Symmetric + Irr-1; black line). No enhancement is seen with respect to the Non-interacting Irr-1 galaxies (green line).

(A color version of this figure is available in the online journal.)

et al. 2008) and in mixed environments (Robaina et al. 2009; Alonso et al. 2004).

While mergers in the A901/902 clusters enhance the SFR of individual galaxies, it is clear that they do not contribute much to the total SFR of the cluster. We compute the SFR density of mergers to non-interacting galaxies in the same volume of A901/902 by taking the ratio of the total SFR in each class. If we include only the 20 distorted mergers in Table 2, we find that the contribution of mergers to the SFR density of the clusters to be $10 \%$. Alternatively, if we include all of the 36 visually classified mergers in Table 2 , before accounting for false projection pairs, we find the contribution of mergers to the SFR density to be $15 \%$. Thus, we find that mergers contribute only a small fraction (between $10 \%$ and 15\%) of the total SFR density of the A901/902 clusters compared to non-interacting galaxies. The small contribution of mergers to the total cluster SFR density is likely due to the low number of mergers in A901/ 902, and the fact that these mergers only cause a modest SFR enhancement.

\section{SUMMARY}

We present a study of the frequency, distribution, color, and SF properties of galaxy mergers in the A901/902 supercluster at $z \sim 0.165$, using a sample of 893 bright $\left(R_{\text {Vega }} \leqslant 24\right)$ intermediate-mass $\left(M_{*} \geqslant 10^{9} M_{\odot}\right)$ galaxies. The sample is complete in stellar mass down to $10^{9} M_{\odot}$. We use HST ACS F606W data from the STAGES survey, COMBO-17, Spitzer $24 \mu \mathrm{m}$, and $X M M$-Newton X-ray data. Our results are as follows:

1. We visually classify the ACS F606W images of the sample galaxies into five main visual classes: Mergers of Types 1,
$2 \mathrm{a}$, and $2 \mathrm{~b}$, and Non-Interacting systems of type Symmetric and Irr-1 (Section 3.2; Tables 1-3; Figures 2, 3, and 5) and obtain a final sample of 886 systems. We classify the systems as mergers if they show evidence of a recent or ongoing merger of mass ratio $>1 / 10$. Mergers of Type 1 essentially appear as an advanced merger remnant, which hosts morphological distortions similar to those produced in simulations of mergers of mass ratio $>1 / 10$. Mergers of Type $2 \mathrm{a}$ and $2 \mathrm{~b}$ appear in ACS images as a very close $(d<10 \mathrm{kpc})$ overlapping pair of two galaxies. The main difference between them is that mergers of Type $2 b$ are resolved into two separate galaxies by the groundbased COMBO-17 data, while mergers of Type $2 \mathrm{a}$ are not.

We perform Monte Carlo simulations to gauge the level of contamination from projection pairs. In the final analysis, we minimize the contamination from false projection pairs by considering only mergers of Type 1 and morphologically distorted systems of Types $2 \mathrm{a}$ and $2 \mathrm{~b}$ as reliable merger candidates. We identify 20 morphologically distorted mergers (13 of Type 1, 3 of Type $2 \mathrm{a}$, and 4 of Type $2 \mathrm{~b}$ ) in the sample. The fraction $f_{\text {merge }}$ of systems with $M_{*} \geqslant 10^{9} M_{\odot}$, which show evidence of a recent or ongoing merger of mass ratio $>1 / 10$, is $0.023 \pm 0.007$. The estimated fractions of likely major mergers, likely minor mergers, and ambiguous cases are $0.01 \pm 0.004(9 / 886), 0.006 \pm 0.003(5 / 886)$, and $0.007 \pm 0.003(6 / 886)$, respectively. Similar merger fractions are obtained among systems with $M_{*} \geqslant 3.0 \times 10^{9} M_{\odot}$.

2. We also estimate the merger fraction using the automated CAS merger criterion $(A>0.35$ and $A>S$; Section 4.2 and Figure 6). Among systems with $M_{*} \geqslant 10^{9} M_{\odot}$, we find the merger fraction to be $\sim 18 / 886$ or $0.02 \pm 0.006$. While the CAS-based merger fraction may not be widely different from the visually based merger fraction, the two methods pick different systems. The CAS criterion only captures 11 of the $20(55 \% \pm 20 \%)$ visually classified mergers. Furthermore, the remaining $7 / 18(39 \% \pm 14 \%)$ of the systems captured by CAS turn out to be non-interacting systems (Table 4). These contaminants tend to be dusty or highly inclined galaxies and systems with low-level asymmetries, which are probably due to SF (Figure 7).

3. We compare our visually based merger fraction in the A901/902 clusters with those reported in other clusters and groups out to $z \sim 0.8$ (Section 4.5 and Figure 13). The low merger fractions among intermediate-mass $\left(10^{9}\right.$ to a few $\left.\times 10^{10} M_{\odot}\right)$ or intermediate luminosities $\left(L<L^{*}\right)$ galaxies in the A901/902 clusters and other low-redshift clusters are consistent with predictions from hierarchical models. However, we cannot yet test the predicted rate of evolution of the merger fraction with redshfit. Data on the merger fraction among $L \leqslant L^{*}$ cluster galaxies, based on our study and three other published studies, allow for a wide spectrum of scenarios, ranging from no evolution to evolution by a factor of $\sim 5$ over $z \sim 0.17$ to 0.4 .

4. Throughout this paper, we consider the core of each cluster to be at a projected clustocentric radius of $R \leqslant 0.25 \mathrm{Mpc}$. We refer to the region between the cluster core and the virial radius as the outer region of the clusters $(0.25 \mathrm{Mpc}<$ $R \leqslant 1.2 \mathrm{Mpc})$. We refer to the region outside the virial radius $(1.2 \mathrm{Mpc}<R \leqslant 2.0 \mathrm{Mpc})$ as the outskirt region of the clusters. The mergers are found to lie outside the cluster core (Section 4.3 and Figure 9), although the timescale for collisions and close encounters is shortest in 


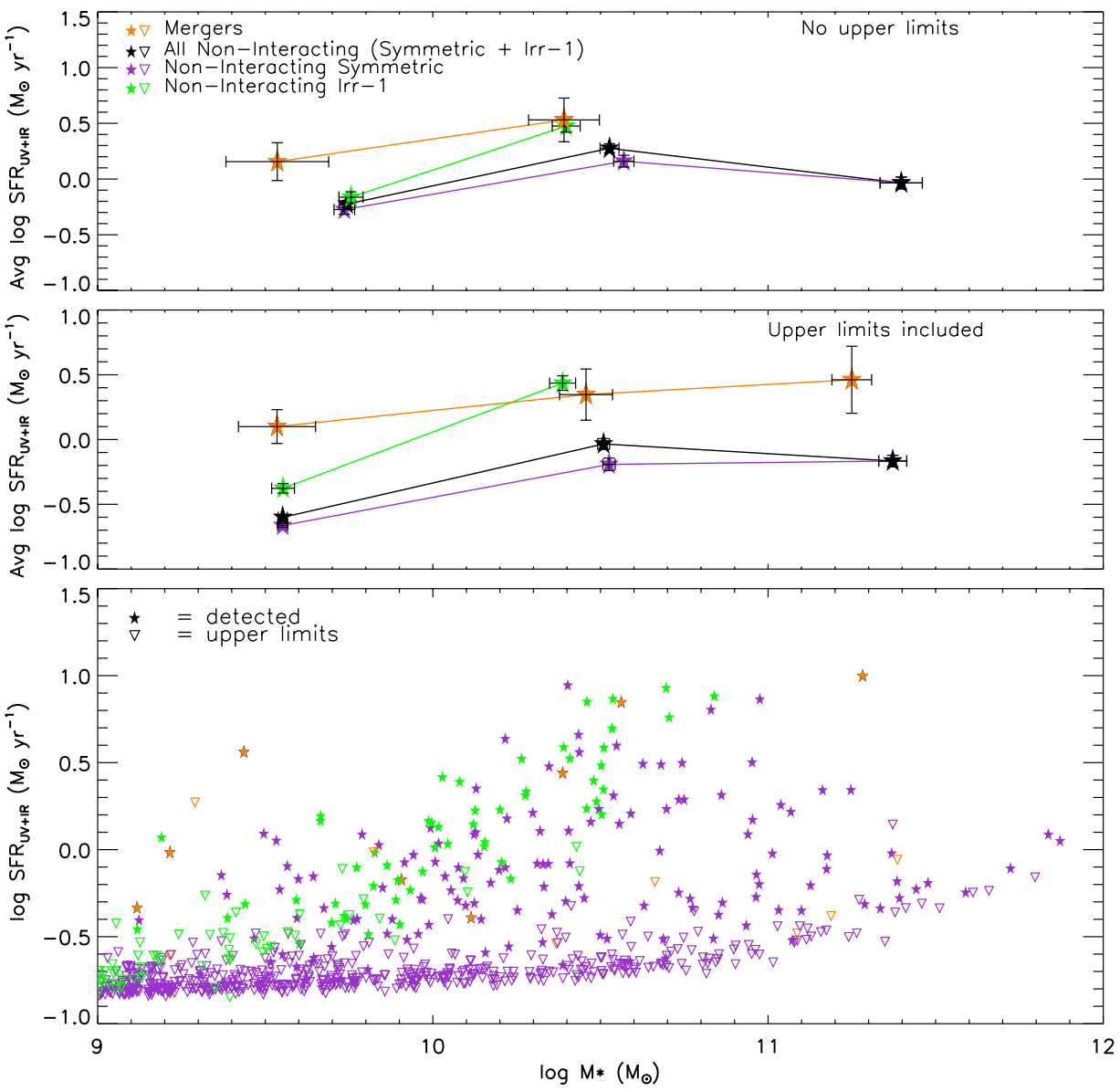

Figure 16. Bottom panel: the UV+IR-based SFR is plotted against stellar mass for the sample of bright intermediate-mass $\left(M_{*} \geqslant 10^{9} M_{\odot}\right)$ systems. Galaxies are coded according to their visual classes: Merger, Non-interacting Irr-1, and Non-interacting Symmetric. We only consider here the 20 distorted mergers in Table 2. For galaxies that were observed and detected at $24 \mu \mathrm{m}$, the UV+IR-based SFR is plotted as stars. For galaxies that were observed but undetected at $24 \mu \mathrm{m}$, we use the detection limit as an upper limit for the UV+IR-based SFR, and plot this limit as inverted triangles. Middle panel: the average UV+IR-based SFR is plotted against stellar mass for galaxies of different visual classes. We include galaxies with a $24 \mu \mathrm{m}$ detection, as well as those with only upper limits on the $24 \mu \mathrm{m}$ flux. Top panel: same as the middle panel, except that we only include galaxies with a $24 \mu \mathrm{m}$ detection and exclude those with upper limits. In both top and middle panels, we find that for the few mergers present (orange line), the average SFR $\mathrm{UV}_{\mathrm{IR}}$ is typically enhanced by only a factor of $\sim 1.5$ compared to the Non-interacting Symmetric galaxies (purple line) and to all Non-interacting galaxies (i.e., Symmetric + Irr-1; black line).

(A color version of this figure is available in the online journal.)

the core $(<1 \mathrm{Gyr})$. We suggest that this is due to the large velocity dispersion $\left(700-900 \mathrm{~km} \mathrm{~s}^{-1}\right)$ of galaxies in the core. Such a dispersion makes it less likely that a close encounter between two galaxies culminates into a merger or a disruptive interaction associated with a large amount of tidal heating.

All of the galaxy mergers lie in the region $(0.25 \mathrm{Mpc}<$ $R \leqslant 2 \mathrm{Mpc}$ ), between the core and cluster outskirt, although the timescale for collisions and close encounters is quite large $(\gg 1 \mathrm{Gyr})$ in this region. One possible explanation might be that the galaxy velocity dispersion drops in the outer region, hence favoring mergers. However, limited number statistics of current spectroscopic data do not allow us to assess this possibility. Another possible scenario is that the interacting galaxies in the outer region and cluster outskirt are part of groups or field galaxies that are being accreted along cosmological filaments by the A901/ 902 clusters. We find that our estimated number density and fraction of mergers to be similar to that predicted at typical group overdensities in $N$-body simulations of groups and field galaxies accreting into the A901/902 clusters (Figure 12). This suggests the ongoing growth of the clusters via accretion of group and field galaxies. The preferentially blue color of the mergers (see below) also supports this scenario.

5. Out of the 20 morphologically distorted merger remnants and merging pairs in the sample, 16 lie on the blue cloud and 4 are in the red sample (Section 4.6 and Figure 14). The fraction of mergers, which lie on the blue cloud is $80 \% \pm 18 \%(16 / 20)$. This is over a factor of 2 higher than the fraction $(294 / 866$ or $34 \% \pm 7 \%)$ of non-interacting galaxies, which lie on the blue cloud, suggesting that mergers are preferentially blue compared to non-interacting galaxies. This effect may be due to the enhanced recent SF in mergers and/or due to the possibility that the mergers are part of group and field galaxies, which are accreting into the cluster.

6. Among intermediate-mass $M_{*} \geqslant 10^{9} M_{\odot}$ systems, the average $\mathrm{SFR}_{\mathrm{UV}}$ and $\mathrm{SFR}_{\mathrm{UV}+\mathrm{IR}}$ of mergers are typically enhanced by only a modest factor of $\sim 2$ (Figure 15) and $\sim 1.5$ (Figure 16), respectively, compared to the noninteracting galaxies (i.e., Symmetric + Irr-1). This modest enhancement is consistent with the theoretical predictions of di Matteo et al. (2007), based on a recent statistical study of several hundred simulated galaxy collisions. Our results of a modest enhancement and a low merger fraction 
culminate in our finding that mergers contribute only a small fraction (between $10 \%$ and $15 \%$ ) of the total SFR density of the A901/902 clusters.

A.H. and S.J. gratefully acknowledge support from NSF grant AST-0607748, LTSA grant NAG5-13063, as well as programs HST-GO-10395, HST-GO-10861, and HST GO-11082, which were supported by NASA through a grant from the Space Telescope Science Institute, which is operated by the Association of Universities for Research in Astronomy, Incorporated, under NASA contract NAS5-26555. We thank Christopher Conselice for his assistance with CAS. E.vK. and M.B. were supported by the Austrian Science Foundation FWF under grant P18416, C.Y.P. by STScI and NRC-HIA Fellowship programmes, C.H. by European Commission Programme Sixth Framework Marie Curie Outgoing International Fellowship under contract MOIFCT-2006-21891 and a CITA National fellowship, M.E.G. and C.W. by an STFC Advanced Fellowship, E.F.B, and K.J. by the DFG's Emmy Noether Programme, A.B. by the DLR (50 OR 0404), S.F.S. by the Spanish MEC grants AYA2005-09413C02-02 and the PAI of the Junta de Andaluc as research group FQM322, L.V.W. by NSERC, ClfAR and CFI, and D.H.M. by NASA under LTSA Grant NAG5-13102. The STAGES team thanks Hans-Walter Rix for providing essential support contributing to the success of the STAGES project. This research made use of NASA's Astrophysics Data System and NASA/ IPAC Extragalactic Database.

\section{REFERENCES}

Abraham, R. G., et al. 1996, ApJS, 107, 1

Alonso, M. S., et al. 2004, MNRAS, 352, 1081

Balogh, M. L., Navarro, J. F., \& Morris, S. L. 2000, ApJ, 540, 113

Balogh, M. L., et al. 1998, ApJ, 504, 75

Balogh, M. L., et al. 1999, ApJ, 527, 54

Balogh, M. L., et al. 2000, MNRAS, 318, 703

Barnes, J. E. 1992, ApJ, 393, 484

Barnes, J. E., \& Hernquist, L. E. 1991, ApJ, 370, 65

Barton, E. J., Geller, M. J., \& Kenyon, S. J. 2000, ApJ, 530, 660

Barton, E. J., Geller, M. J., \& Kenyon, S. J. 2003, ApJ, 582, 668

Bell, E., et al. 2005, ApJ, 625, 23

Bell, E., et al. 2007, ApJ, 663, 834

Bershady, M. A., Jangren, J. A., \& Conselice, C. J. 2000, AJ, 119, 2645

Bertin, E., \& Arnouts, S. 1996, A\&AS, 117, 393

Binggeli, B., Sandage, A., \& Tammann, G.A. 1988, ARA\&A, 26, 509

Binggeli, B., Tammann, G. A., \& Sandage, A. 1987, AJ, 94, 251

Binney, J., \& Tremaine, S. 1987, Galactic Dynamics (Princeton: Princeton Univ. Press)

Blanton, M. R., et al. 2005, ApJ, 629, 143

Borch, A., et al. 2006, A\&A, 453, 869

Butcher, H., \& Oemler, A. Jr. 1978, ApJ, 219, 18

Caldwell, J. A. R., et al. 2008, ApJS, 174, 136

Carlberg, R. G., et al. 1997, ApJ, 476L, 7C

Chabrier, G. 2003, PASP, 115, 763

Cole, S., et al. 2000, MNRAS, 319, 168

Cole, S., et al. 2001, MNRAS, 326, 255

Conselice, C. J. 2003, AJ, 126, 1183

Conselice, C. J. 2006, ApJ, 638, 686

Conselice, C. J., Bershady, M. A., \& Jangren, A. 2000, ApJ, 529, 886

Couch, W., et al. 1998, ApJ, 497, 188

de Propis, R., et al. 2003, MNRAS, 345, 725

den Hartog, R., \& Katgert, P. 1996, MNRAS, 279, 349

Diaferio, A., et al. 2001, MNRAS, 323, 999

di Matteo, P., et al. 2007, A\&A, 468, 61
Dressler, A. 1980, ApJ, 236, 351

Dressler, A., et al. 1994, ApJ, 435, 23

Dressler, A., et al. 1997, ApJ, 490, 577

Ellison, S. L., et al. 2008, AJ, 135, 1877

Gavazzi, G., \& Jaffe, W. 1985, ApJ, 294, 89

Gilmour, R., et al. 2007, MNRAS, 380, 1467

Giovanelli, R., Haynes, M. P., \& Chincarini, G. L. 1986, ApJ, 300, 77

Gnedin, O. Y. 2003, ApJ, 582, 141

Gottloeber, S., Klypin, A., \& Kravtsov, A. V. 2001, ApJ, 546, 223

Gray, M. E., et al. 2002, ApJ, 568, 141

Gray, M. E., et al. 2009, MNRAS, 393, 1275

Gunn, J. E., \& Gott, J. R., III. 1972, ApJ, 176, 1

Hernquist, L. E. \& Mihos 1995, ApJ, 448, 41

Heymans, C., et al. 2008, MNRAS, 385, 1431

Hopkins, P. F., Cox, T. J., Younger, J. D., \& Hernquist, L. 2009, ApJ, 691, 1168

Jogee, S. 2006, in Physics of Active Galactic Nuclei at all Scales, Lecture Notes in Physics, Vol. 693, ed. D. Alloin, R. Johnson, \& P. Lira (Springer: Berlin), 143

Jogee, S., Kenney, J. D. P., \& Smith, B. J. 1999, ApJ, 526, 665

Jogee, S., Miller, S., Penner, K., Skelton, R. E., Conselice, C. J., Somerville, R. S., \& GEMS Collaboration, 2009, ApJ, 697, 1971

Jogee, S., et al. 2008, in ASP Conf. Ser. 396, Formation and Evolution of Galaxy Disks, ed. J. G. Funes \& S. J. E. M. Corsini (San Francisco, CA: ASP), 337

Kartaltepe, J. S., et al. 2007, ApJS, 172, 320

Kennicutt, R. C. Jr. 1983, AJ, 88, 1094

Kennicutt, R. C. Jr. 1998, ARA\&A, 36, 189

Khochfar, S., \& Burkert, A. 2001, ApJ, 561, 517

Koopmann, R. A., \& Kenney, J. D. P. 2004, ApJ, 613, 866

Kroupa, P. 2001, MNRAS, 322, 231

Kroupa, P., Tout, C. A., \& Gilmore, G. 1993, MNRAS, 262, 545

Larson, R. B., Tinsley, B. M., \& Caldwell, C. N. 1980, ApJ, 237, 692

Lavery, R. J., \& Henry, J. P. 1988, ApJ, 330, 596

Lavery, R. J., et al. 1992, AJ, 104, 2067

Lin, L., et al. 2004, ApJL, 617, 9

Lotz, J., et al. 2008, MNRAS, 391, 1137

Margoniner, V. E., et al. 2001, ApJ, 548, 143

McGee, L. M., et al. 2008, MNRAS, 387, 1065

McIntosh, D. H., et al. 2008, MNRAS, 388, 1537

Mihos, J. C., \& Hernquist, L. 1996, ApJ, 464, 641

Mihos, J. C., et al. 1995, ApJ, 438, 75

Miller, S., et al. 2008, in ASP Conf. Ser. 393, 'Frank N. Bash Symp. 2007: New Horizons in Astronomy,' ed. A. Frebel, J. Maund, J. Shen, \& M. Siegel (San Francisco, CA: ASP), 235

Moore, B., et al. 1998, ApJ, 495, 139

Moore, B., et al. 1996, Nature, 379, 613

Naab, T., \& Burkert, A. 2001, ApJ, 555, 91

Negroponte, J., \& White, S. D. M. 1983, MNRAS, 205, 1009

Oemler, A., Jr., et al. 1997, ApJ, 474, 561

Papovich, C., \& Bell, E. F. 2002, ApJ, 579, L1

Postman, M., \& Geller, M. J. 1984, ApJ, 281, 95

Quilis, V., Moore, B., \& Bower, R. 2000, Science, 288, 1617

Quinn, P. J., Hernquist, L., \& Fullagar, D. P. 1993, 403, 74

Robaina, A., et al. 2009, ApJ, 704, 324

Robertson, B., et al. 2004, ApJ, 606, 32

Smith, B. J. 1997, AJ, 114, 138

Steinmetz, M., \& Navarro, J. F. 2002, New Astron., 7, 155

Struck, C. 1997, ApJS, 113, 269

Tago, E., et al. 2008, A\&A, 479, 927

The, L. S., \& White, S. D. M. 1986, AJ, 92, 1248

Toomre, A., \& Toomre, J. 1972, ApJ, 178, 623

Tran, K.-V. H., et al. 2005, ApJ, 627, L25

Tran, K.-V. H., et al. 2008, ApJ, 683, 17

van Dokkum, P. G., et al. 1998, ApJ, 500, 714

van Dokkum, P. G., et al. 1999, ApJ, 520, L95

van Kampen, E., \& Katgert, P. 1997, MNRAS, 289, 327

Whitmore, B., et al. 1993, ApJ, 407, 489

Wolf, C., Gray, M. E., \& Meisenheimer, K. 2005, A\&A, 443 , 435

Wolf, C., et al. 2004, A\&A, 421,913

Wolf, C., et al. 2009, MNRAS, 393, 1302

Zabludoff, A. I., \& Franx, M. 1993, AJ, 106, 1314

Zepf, S. E. 1993, ApJ, 407, 448 